\title{
STELLAR ARCHAEOLOGY: A KECK PILOT PROGRAM ON EXTREMELY METAL-POOR STARS FROM THE HAMBURG/ESO SURVEY. II. ABUNDANCE ANALYSIS ${ }^{1}$
}

\author{
Eugenio Carretta, ${ }^{2}$ Raffaele Gratton, ${ }^{2}$ Judith G. Cohen, ${ }^{3}$ Timothy C. Beers, ${ }^{4}$ and Norbert Christlieb ${ }^{5}$ \\ Received 2001 October 1; accepted 2002 March 26
}

\begin{abstract}
We present a detailed abundance analysis of eight stars selected as extremely metal-poor candidates from the Hamburg/ESO Survey (HES). For comparison, we have also analyzed three extremely metal-poor candidates from the HK survey, and three additional bright metal-poor stars. With this work, we have doubled the number of extremely metal-poor stars $([\mathrm{Fe} / \mathrm{H}] \leq 3.0 \mathrm{dex})$ with high-precision abundance analyses. Based on this analysis, our sample of extremely metal-poor candidates from the HES contains three stars with $[\mathrm{Fe} / \mathrm{H}] \leq-3.0$ dex, three more with $[\mathrm{Fe} / \mathrm{H}] \leq-2.8$ dex, and two stars that are only slightly more metal-rich. Thus, the chain of procedures that led to the selection of these stars from the HES successfully provides a high fraction of extremely metal-poor stars. We verify that our choices for stellar parameters, derived in Paper I and independently of the high-dispersion spectroscopic analysis, lead to acceptable ionization and excitation balances for Fe. Substantial non-LTE effects in Fe appear to be ruled out by the above agreement, even at these extremely low metallicities. For the $\alpha$-elements $\mathrm{Mg}, \mathrm{Si}, \mathrm{Ca}$, and $\mathrm{Ti}$, the light element $\mathrm{Al}$, the iron-peak elements $\mathrm{Sc}, \mathrm{Cr}$, and $\mathrm{Mn}$, and the neutron-capture elements $\mathrm{Sr}$ and $\mathrm{Ba}$, we find trends in abundance ratios $[\mathrm{X} / \mathrm{Fe}]$ similar to those found by previous investigations. These trends appear to be identical for giants and for dwarfs. However, the scatter in most of these ratios, even at $[\mathrm{Fe} / \mathrm{H}] \leq-3.0$ dex, is surprisingly small. Only $\mathrm{Sr}$ and $\mathrm{Ba}$, among the elements we examined, show scatter larger than the expected errors. Future work (the "0Z Project") will provide much stronger constraints on the scatter (or lack thereof) in elemental abundances for a substantially greater number of stars. We discuss the implications of these results for the early chemical evolution of the Galaxy, including such issues as the number of contributing supernovae and the sizes of typical protogalactic fragments in which they were born. In addition, we have identified a very metalpoor star in our sample that appears to represent the result of the $s$-process chain, operating in a very metalpoor environment, and exhibits extremely enhanced $\mathrm{C}, \mathrm{Ba}$, and $\mathrm{Pb}$ and somewhat enhanced $\mathrm{Sr}$.
\end{abstract}

Key words: Galaxy: evolution — Galaxy: formation — Galaxy: halo — Galaxy: stellar content — stars: abundances

On-line material: machine-readable tables

\section{INTRODUCTION}

Stellar archaeology is the study of present stellar generations in order to infer the characteristics of a previous stellar generation that no longer exists. This is one of the primary aims for investigations of the chemical composition of extremely metal-poor Population II stars, as they provide important clues to the properties (e.g., mass, composition) of the very first objects formed in the Galaxy, the so-called Population III stars.

Long-lived, slowly evolving main-sequence dwarfs are quite suitable for this purpose, since they retain in their atmospheres the elements produced by previously born massive stars that exploded as Type II supernovae (SNe). Unlike the stars presently in the giant branch stage of evolution, main-sequence stars are expected to be unaffected by internal mixing during their lifetimes (although, in some

\footnotetext{
${ }^{1}$ Based in large part on observations obtained at the W. M. Keck Observatory, which is operated jointly by the California Institute of Technology, the University of California, and NASA.

2 Osservatorio Astronomico di Padova, Instituto Nazionale di Astrofisica, vicolo dell'Osservatorio 5, I-35122 Padova, Italy.

${ }^{3}$ Palomar Observatory, Mail Stop 105-24, California Institute of Technology, 1201 East California Boulevard, Pasadena, CA 91125.

${ }^{4}$ Department of Physics and Astronomy, Michigan State University, East Lansing, MI 48824-1116.

${ }^{5}$ Hamburger Sternwarte, Gojenbergsweg 112, D-21029 Hamburg, Germany.
}

cases, they may exhibit the spectral signatures of contamination from material transferred from close, evolved, companions).

Here we adopt the definition given in Paper I (Cohen et al. 2002), and we consider only stars with $[\mathrm{Fe} / \mathrm{H}] \leq-3$ dex to be extremely metal-poor (EMP). ${ }^{6}$ This definition seems almost straightforward, since all previous investigations (see the review by McWilliam 1997) have revealed that at $[\mathrm{Fe} / \mathrm{H}]=-2.4$ dex, many elemental ratios $[\mathrm{X} / \mathrm{Fe}]$ (where $\mathrm{X}$ is $\mathrm{Ba}, \mathrm{Sr}, \mathrm{Cr}, \mathrm{Al}$, or $\mathrm{Mn}$ ) display a sudden change in the slope of the relationship of $[\mathrm{X} / \mathrm{Fe}]$ versus $[\mathrm{Fe} / \mathrm{H}]$. McWilliam et al. (1995, hereafter McW95) and others have since suggested that the patterns observed at very low metallicity can be explained by assuming that a stochastic mechanism is at work, with only a few SNe responsible for the observed enrichment patterns. By selecting those stars with $[\mathrm{Fe} / \mathrm{H}] \leq-3$ dex, we can be certain that we are sampling a regime where stars were polluted by the very first $\mathrm{SNe}$, in an environment likely to have been rather different from that in which the bulk of Galactic stars formed.

The literature concerning EMP stars is continuously increasing, as ever more efficient spectrographs at large tele-

\footnotetext{
${ }^{6}$ We use the usual spectroscopic notation: $\log n(\mathrm{~A})$ is the abundance (by number) of the element $\mathrm{A}$ in the usual scale where $\log n(\mathrm{H})=12$, while $[\mathrm{A} / \mathrm{H}]$ denotes the logarithmic ratio of the abundances of elements $\mathrm{A}$ and $\mathrm{H}$ in the star, minus the same quantity in the Sun.
} 
scopes come on-line, analysis techniques are refined, and laboratory measurements of fundamental atomic parameters required for detailed abundance analyses are carried out. Interest in this area of astrophysics arises for a number of reasons, since the study of these objects provides insights into such relevant issues as the early chemical evolution of the Galaxy, nucleosynthesis by zero-metallicity massive stars, and the role of the $r$-process and the $s$-process in building up the presently observed abundances of neutroncapture elements in stars. Theories of the nucleosynthetic mechanisms themselves benefit from direct comparison with observed abundance ratios in EMP stars, in order to tune model yields. On the other hand, by use of the predicted yields from $\mathrm{SNe}$ of different masses, one might attempt to decode the observed run of abundances as a function of metallicity, in order to derive the range of numbers and masses of SNe contributing to the chemical enrichment in various environments, as well as the epochs of the building up of the Galactic elements (see, e.g., Karlsson \& Gustafsson 2001).

Moreover, a direct link to the distant universe is provided by dating methods that use cosmochronology (age estimates based on the radioactive decay of unstable heavy nuclei in EMP stars) to provide independent measurements of the ages of the oldest stars in our Galaxy (Sneden et al. 2000; Cayrel et al. 2001a, 2001b; Toenjes et al. 2001), which can be compared with the ages of other apparently primordial objects, such as globular clusters, derived by different methods (see Carretta et al. 2000 and references therein).

The shortcoming, up to now, has been the small size of available stellar samples, due to the relative rarity of EMP stars, their faint apparent magnitudes, and the need for high-resolution, high signal-to-noise ratio $(\mathrm{S} / \mathrm{N})$ spectroscopy to derive their elemental abundances with suitable precision. The presently available sample of such stars is simply too small for statistical studies that might provide strong constraints on the aforementioned problems. In fact, summing up all previous high-dispersion analyses of very metalpoor stars (those with $[\mathrm{Fe} / \mathrm{H}] \leq-2.0 \mathrm{dex}$ ), the total sample with published detailed analyses hardly reaches 50 objects.

We are mainly interested in studying the mechanisms involved in the early chemical evolution of the Galaxy. The large intrinsic spread in (some) elemental ratios found at extremely low metallicities requires a very large database, not only to properly sample the observed trends, but, more importantly, to quantify the scatter in the observed elemental abundance distributions as a function of declining metallicity. An increase of available sample sizes for EMP stars by an order of magnitude is required to fully understand the nature of the very first generations of Galactic stars.

In our ongoing study, we intend to exploit the recently completed Hamburg/ESO Survey (HES; Wisotzki et al. 1996; Christlieb et al. 2001a, 2001b) to significantly increase the number of EMP candidates with available high-S $/ \mathrm{N}$, high-dispersion spectroscopy. Herein we present the results of the Keck pilot program on EMP stars, in which we test the ability of the HES to deliver a large sample of newly identified EMP stars for abundance analysis.

The selection, observations, and data reduction of the present sample are discussed at length in Paper I; the present paper will deal only with the abundance analysis. In $\S 2$, there is a brief summary of relevant information given in Paper I. The equivalent width measurements and tests of their quality are described in $\S 3$. The derived abundances are presented in $\S 4$ and discussed in $\S 5$. The last section summarizes our current results.

\section{OBSERVATIONS, DATA REDUCTION, AND ATMOSPHERIC PARAMETERS}

The selection, observational details, and the preliminary data reduction of our program stars are discussed in Paper I. Here we briefly summarize the essential information.

Eight candidate EMP stars from the HES were observed with the HIRES spectrograph (Vogt et al. 1994) at Keck I on two nights in 2000 September. On the same nights, we also acquired spectra for three EMP candidates from the HK survey (Beers, Preston, \& Shectman 1985, 1992), as well as three well-studied bright metal-poor stars as comparisons. One of the HES stars turned out to be a rediscovery of a star from the HK survey (HE 2344-2800 = CS 22966045). The relevant parameters of the observations, as well as photometry and the adopted data analysis for all stars in our sample, are given in Table 1 of Paper I.

A spectral resolving power of $R=45,000$ was used with a 0 ".86 slit projecting to 3 pixels in the HIRES focal-plane CCD detector, resulting in spectra covering the region from 3870 to $5400 \AA$, with essentially no gaps. The figure of merit, $F$, as defined by Norris, Ryan, \& Beers (2001, hereafter NRB01), is $\sim 600$ for the worst of our spectra, which guarantees the high quality of our observational material. In this paper, we have doubled the sample of high-precision $(F>600)$ spectra available for stars with $[\mathrm{Fe} / \mathrm{H}]<-3.0$ dex, including three newly discovered EMP stars. Some examples of the spectra are shown in Paper I.

\subsection{Adopted Atmospheric Parameters}

The procedure used to derive $T_{\text {eff }}$ estimates for our program stars is fully explained in $\S \S 4-6$ of Paper I. Very briefly, $T_{\text {eff }}$ is derived from broadband colors, taking the mean estimates deduced from the dereddened $V-K$ and $V-J$ colors. We used the grid of predicted broadband colors and bolometric corrections of Houdashelt, Bell, \& Sweigart (2000), based on the MARCS stellar atmosphere code (Gustafsson et al. 1975), and corrected the colors for reddening by adoption of the extinction maps of Schlegel, Finkbeiner, \& Davis (1998) (see Table 1 in Paper I).

With $T_{\text {eff }}$ fixed, the gravity $\log g$ was obtained using the $\mathrm{Y}^{2}$ isochrones (Yi et al. 2001); we adopted the $14 \mathrm{Gyr}$, $[\mathrm{Fe} / \mathrm{H}]=-3.3$ isochrone. For the star HD 140283, we adopted the $\log g$ obtained by Korn \& Gehren (2000), derived from the Hipparcos parallax.

Holding $T_{\text {eff }}$ and $\log g$ fixed, the final overall metallicities $[\mathrm{A} / \mathrm{H}]$ for the stars were obtained iteratively, by matching observed equivalent widths (EWs) with the synthetic ones computed by integrating the equation of transport at different wavelengths along each line for the flux, extracted from a model atmosphere in the grid of Kurucz (1993a), with no overshooting. ${ }^{7}$ In fact, Castelli, Gratton, \& Kurucz (1997) noted that Kurucz models with the convective overshooting option switched off better reproduce observables in stars other than the Sun. Microturbulent velocities $v_{t}$ were

\footnotetext{
${ }^{7}$ Models are interpolated linearly in $T_{\text {eff }}$ and logarithmically in the other quantities. Note that model atmospheres for stars with $[\mathrm{Fe} / \mathrm{H}]<-3$ dex are not interpolated but extrapolated, since the grid of Kurucz does not have models below this metallicity.
} 
TABLE 1

Adopted Model Atmosphere Parameters

\begin{tabular}{|c|c|c|c|c|c|c|}
\hline Star & $\begin{array}{l}T_{\text {eff }} \\
(\mathrm{K})\end{array}$ & $\begin{array}{l}\log g \\
(\operatorname{dex})\end{array}$ & $\begin{array}{c}{[\mathrm{A} / \mathrm{H}]} \\
(\mathrm{dex})\end{array}$ & $\begin{array}{c}v_{t} \\
\left(\mathrm{~km} \mathrm{~s}^{-1}\right)\end{array}$ & $\begin{array}{l}\Delta[\mathrm{Fe} / \mathrm{H}]_{\mathrm{II}-\mathrm{I}} \\
\quad(\mathrm{dex})\end{array}$ & $\begin{array}{c}\Delta(\mathrm{Fe} \mathrm{I} / \chi) \\
\left(\operatorname{dex~eV}^{-1}\right)\end{array}$ \\
\hline HD $140283 \ldots \ldots \ldots \ldots . . . .$. & 5750 & 3.67 & -2.44 & 1.10 & -0.025 & -0.064 \\
\hline $\mathrm{BD}+3^{\circ} 740 \ldots \ldots \ldots \ldots$ & 6355 & 4.00 & -2.70 & 1.42 & -0.005 & -0.063 \\
\hline G139-8 .................... & 6200 & 4.50 & -2.06 & 1.02 & -0.116 & -0.074 \\
\hline BS 17447-029 .......... & 6530 & 4.40 & -2.93 & 1.94 & -0.268 & -0.041 \\
\hline CS 22950-046 ............ & 4730 & 1.30 & -3.30 & 2.02 & -0.170 & -0.072 \\
\hline CS 22878-101 .......... & 4775 & 1.30 & -3.09 & 2.01 & -0.053 & -0.058 \\
\hline HE $2133-1426 \ldots \ldots .$. & 6300 & 4.10 & -2.83 & 1.31 & -0.138 & -0.045 \\
\hline HE $2344-2800 \ldots \ldots \ldots$ & 6625 & 4.30 & -2.56 & 1.42 & 0.036 & -0.043 \\
\hline HE $0024-2523 \ldots \ldots . .$. & 6625 & 4.30 & -2.65 & 0.58 & -0.005 & -0.013 \\
\hline HE $0130-2303 \ldots \ldots . .$. & 6560 & 4.30 & -2.96 & 1.32 & -0.087 & -0.017 \\
\hline HE $0132-2429 \ldots \ldots \ldots$ & 5310 & 3.40 & -3.59 & 1.28 & -0.217 & -0.146 \\
\hline HE $0148-2611 \ldots \ldots .$. & 6550 & 4.30 & -2.98 & 0.78 & -0.309 & 0.002 \\
\hline HE $0218-2738 \ldots \ldots . .$. & 6550 & 4.30 & -3.54 & 0.84 & $\ldots$ & -0.077 \\
\hline HE $0242-0732 \ldots \ldots .$. & $6360^{\mathrm{a}}$ & $4.40^{\mathrm{a}}$ & -3.21 & 0.40 & -0.212 & -0.150 \\
\hline
\end{tabular}

a Value slightly modified from that of Paper I; see text for details.

derived by eliminating any trend in derived abundances of Fe I lines with the expected EWs (see Magain 1984). The adopted atmospheric parameters are summarized in Table 1.

\section{EQUIVALENT WIDTHS}

Equivalent widths were measured from the one-dimensional, normalized spectra using an automatic routine that determines a local continuum level for each line by an iterative clipping procedure. A fraction $C$ of the 200 spectral pixels centered on the line to be measured is used; the highest $200 C$ pixels are the initial data set for this process. After various tests, we adopted $C=1$ for the spectra of all stars, except the three very bright stars and the two giants from the HK survey. For these stars, $C=0.5$ was used.

The lines were then measured by a Gaussian fitting routine using a small spectral region (of width 1.6 times the FWHM) centered on their expected location, based on a preliminary determination of the geocentric radial velocity. A number of lines were discarded at this point based on several criteria (features that were not well centered, that were either too broad or too narrow, etc.). After this first measurement, a relation was set between equivalent width and FWHM for each spectrum, examples of which are shown in Figure 1. This relation was then used to obtain a better determination for each absorption line, invoking a different Gaussian fitting routine. This second routine has only one free parameter for each line, effectively the central depth of the line profile, because the line center's location is fixed by the average radial velocity determined from all the lines measured in the second step. Again, several criteria were used to discard lines at this point in the analysis (e.g., asymmetric error distributions, indicating lines that are not well centered; large residuals compared with expected noise). These procedures allow us to obtain very stable measures of the EWs, with random errors close to those expected from photon noise statistics (Cayrel 1989). Of course, systematic errors may still be present, in particular those related to the adopted reference continua and the relation between equivalent width and FWHM.

Tables 2 and 3 list the final values of the EWs, along with the adopted atomic parameters for all lines in our list. Table
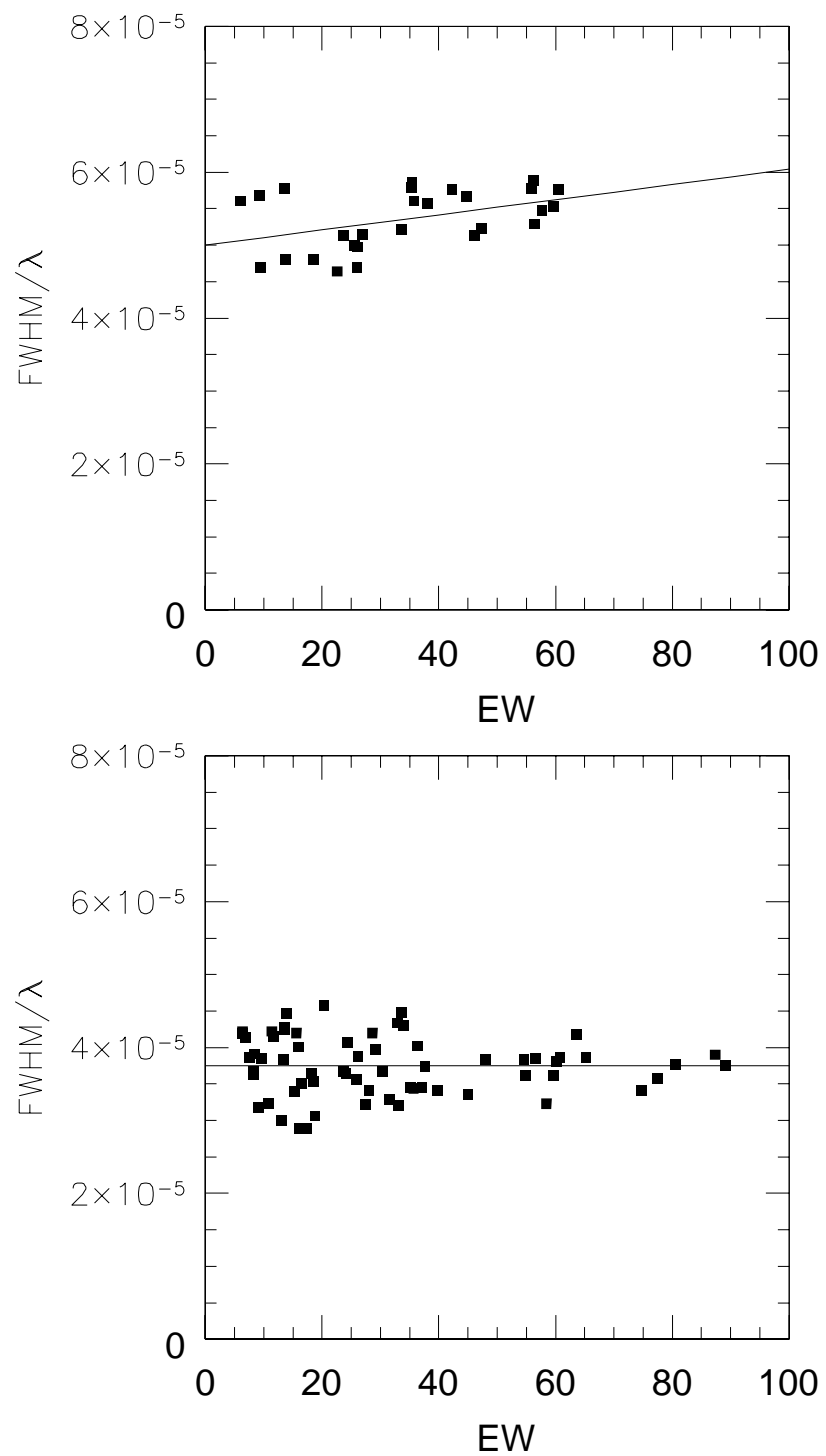

FIG. 1.-Fiducial relation between the FWHM of lines and their EWs for stars HE 0024-2523 (top) and HE 0130-2303 (bottom). 
TABLE 2

Equivalent Widths For Bright STARS AND HK Stars

\begin{tabular}{|c|c|c|c|c|c|c|c|c|c|c|}
\hline Ion & $\begin{array}{c}\lambda \\
(\AA)\end{array}$ & $\begin{array}{c}\chi \\
(\mathrm{eV})\end{array}$ & $\log g f$ & Source & $\begin{array}{l}\text { HD } 140283 \\
(\mathrm{~m} \AA)\end{array}$ & $\begin{array}{c}\mathrm{BD}+3^{\circ} 740 \\
(\mathrm{~m} \AA)\end{array}$ & $\begin{array}{c}\text { G139-8 } \\
(\mathrm{m} \AA)\end{array}$ & $\begin{array}{c}\text { BS 17447-029 } \\
(\mathrm{m \AA})\end{array}$ & $\begin{array}{c}\text { CS } 22950-046 \\
(\mathrm{~m} \AA)\end{array}$ & $\begin{array}{c}\text { CS 22878-101 } \\
(\mathrm{m \AA})\end{array}$ \\
\hline $\operatorname{Mg}{ }_{1} \ldots \ldots . .$. & 4057.52 & 4.34 & -1.20 & 1 & 18.0 & $\ldots$ & 13.3 & $\ldots$ & 11.2 & 21.2 \\
\hline $\operatorname{Mg}_{\mathrm{I}} \ldots \ldots .$. & 4703.00 & 4.34 & -0.67 & 1 & 41.2 & 19.6 & 30.1 & $\ldots$ & 22.8 & 47.0 \\
\hline $\operatorname{Mg}_{\mathrm{I}} \ldots \ldots .$. & 5172.70 & 2.71 & -0.38 & 1 & 139.0 & 101.2 & 126.0 & 81.3 & 130.2 & 150.0 \\
\hline $\operatorname{Mg}_{\mathrm{I}} \ldots \ldots .$. & 5183.62 & 2.72 & -0.16 & 1 & 156.3 & 115.2 & 138.0 & 93.7 & 145.5 & 187.0 \\
\hline Si I........... & 3905.53 & 1.91 & -1.04 & 3 & 125.0 & $\ldots$ & 116.6 & $\ldots$ & 136.9 & 143.9 \\
\hline
\end{tabular}

NoTE.-Table 2 is presented in its entirety in the electronic edition of the Astronomical Journal. A portion is shown here for guidance regarding its form and content.

8 in the Appendix summarizes the comparison between our adopted $g f$ 's and the compilation in the NIST database.

In order to evaluate the internal errors in our measurement of EWs, we compare values obtained for two stars in our sample with similar physical parameters. We performed this comparison using two dwarfs [HE 0130-2303, with $\left(T_{\text {eff }}, \log g,[\mathrm{Fe} / \mathrm{H}], v_{t}\right)=(6560,4.3,-2.96,1.39)$, and $\mathrm{HE}$ $0148-2611$, with $\left(T_{\text {effi }}, \log g,[\mathrm{Fe} / \mathrm{H}], v_{t}\right)=(6550,4.3,-3.07$, 1.25), respectively] and two giants [CS 22950-046: ( $T_{\text {eff }}$, $\left.\log g,[\mathrm{Fe} / \mathrm{H}], v_{t}\right)=(4730,1.3,-3.30,2.02)$; and CS $22878-$ 101: $\left.\left(T_{\text {eff }}, \log g,[\mathrm{Fe} / \mathrm{H}], v_{t}\right)=(4775,1.3,-3.09,2.01)\right]$.

For the two dwarfs, the rms scatter about the regression line between the sets of EWs (see Fig. $2 b$ ) is $4.5 \mathrm{~mA}$. If we assume that both sets of EWs have equal errors, we can estimate typical errors of $3.2 \mathrm{~mA}$ in the EWs. For the giants (Fig. 2a), the rms scatter is $7.9 \mathrm{m \AA}$, corresponding to an error of $5.6 \mathrm{~mA}$ in the EWs for each star. In both cases, these errors are in good agreement with the predicted errors obtained from the formulae derived by Cayrel (1989), given the spectral resolution and the $\mathrm{S} / \mathrm{N}$ characteristics of our data. This confirms the high quality of the spectra and suggests that no extra sources of noise were introduced by the EW extraction procedure.

An external comparison of our derived EWs for stars in our sample can be carried out using the bright, well-studied, metal-poor stars, as well as the stars selected from HK survey.

HD 140283 is the star with the largest number of entries in the Cayrel de Strobel, Soubiran, \& Ralite (2001) catalog (note that our data for this star are of higher quality than those for our typical program stars). Among the large list of previous analyses of this star, we considered three sets of high-quality EWs: Zhao \& Magain (1990; $R \sim 20,000$, $\mathrm{S} / \mathrm{N} \sim 100)$, Gratton \& Sneden (1994; $R \sim 50,000$, $\mathrm{S} / \mathrm{N} \geq 150$ ), and Ryan, Norris, \& Beers (1996, hereafter $\mathrm{RNB} 96 ; R \sim 40,000, \mathrm{~S} / \mathrm{N} \sim 45$ ). The comparison is shown in the three panels of Figure 3, where the one-to-one correspondence lines are also displayed. The linear regression lines are $\mathrm{EW}_{\mathrm{us}}=0.6 \mathrm{m \AA}+(0.94 \pm 0.01) \mathrm{EW}_{\mathrm{ZM} 90}$, with $\sigma=2.2 \AA$ from 59 lines in common, $\mathrm{EW}_{\mathrm{us}}=-1.4$ $\mathrm{m} \AA+(1.04 \pm 0.01) \mathrm{EW}_{\mathrm{GS} 94}$, with $\sigma=2.3 \mathrm{~m} \AA$ from 18 lines in common, and $\mathrm{EW}_{\text {us }}=1.22 \mathrm{~mA}+(0.93 \pm 0.01) \times$ $\mathrm{EW}_{\mathrm{RNB} 96}$, with $\sigma=2.3 \mathrm{~mA}$ from 74 lines in common. The very small scatter present in these comparisons again agrees well with the theoretically predicted errors and confirms the error estimates given above.
For the two giants from the HK survey in common with the McW95 sample, the linear regression between our measurements and their $\mathrm{EWs}$ is $\mathrm{EW}_{\mathrm{McW} 95}=1.6 \mathrm{m \AA}+$ $(1.04 \pm 0.03) \mathrm{EW}_{\text {us }}$, with $\sigma=12.0 \mathrm{~m} \AA$ from 153 lines in the two stars. The McW95 EWs are on average larger than ours (the average offset is $4.1 \mathrm{~mA}$ ); the difference increases for stronger lines. The rather large scatter is most likely due to their measurement errors, arising from spectra having a lower resolving power $(R \sim 22,000)$ and a lower average $\mathrm{S} / \mathrm{N}$ (typically $30-40$ ) than ours.

\section{ABUNDANCE ANALYSIS}

Model atmospheres with appropriate parameters (see Table 1) were extracted from the grid of Kurucz (1993a) with the overshooting option switched off, interpolating among the nearest models in the Kurucz grid. The abundance analysis was performed using measured EWs (Tables 2 and 3); the resulting abundances and elemental ratios for each species in each star are listed in Tables 4 and 5 and are discussed below. In these tables, $n$ is the number of lines used in the analysis of a given ion and $\sigma$ is the rms scatter in abundance from individual lines for the set of lines used for a particular ion.

The abundances of neutral species are computed with respect to $\mathrm{Fe}$ I, while singly ionized species are compared with $\mathrm{Fe}$ II abundances to decrease uncertainties due to the choice of atmospheric parameters. Table 6 shows how the choice of a given set of atmospheric parameters might affect the derived abundances. Values in this table are computed by varying, one at a time, the individual atmospheric parameters and comparing the resulting abundances with the original values. The amount of the variation is set by the uncertainties established for each of the parameters. This exercise was carried out for a giant (CS 22950-046) and for a dwarf (HE 2344-2800), in order to span the whole range of $T_{\text {eff }}$ sampled by program stars. In each case, the first four columns show the sensitivity of abundance ratios to changes in each adopted parameter.

The last column of Table 6 lists for each star the sums (in quadrature) of contributions due to the individual parameters; this provides an estimate of the overall uncertainty in abundance for each species arising from errors in the adopted atmospheric parameters. From Paper I, we derive estimates of our internal errors of about $\pm 100 \mathrm{~K}$ and \pm 0.2 0.3 dex for $T_{\text {eff }}$ and for $\log g$, respectively. In evaluating the 
TABLE 3

EQUivalent WidThS For THE HES STARS

\begin{tabular}{|c|c|c|c|c|c|c|c|c|c|c|c|c|}
\hline Ion & $\left(\begin{array}{l}\lambda \\
(\AA)\end{array}\right.$ & $\begin{array}{c}\chi \\
(\mathrm{eV})\end{array}$ & $\log g f$ & Source & $\begin{array}{c}\text { HE } 2133-1426 \\
(\mathrm{m \AA})\end{array}$ & $\begin{array}{c}\text { HE 2344-2800 } \\
(\mathrm{m} \AA)\end{array}$ & $\begin{array}{c}\text { HE } 0024-2523 \\
(\mathrm{~m} \AA)\end{array}$ & $\begin{array}{c}\text { HE } 0130-2303 \\
(\mathrm{~m} \AA)\end{array}$ & $\begin{array}{c}\text { HE } 0132-2429 \\
(\mathrm{m \AA})\end{array}$ & $\begin{array}{c}\text { HE } 0148-2611 \\
(\mathrm{~m} \AA)\end{array}$ & $\begin{array}{c}\text { HE } 0218-2738 \\
(\mathrm{~m} \AA)\end{array}$ & $\begin{array}{c}\text { HE } 0242-0732 \\
(\mathrm{~m} \AA)\end{array}$ \\
\hline $\operatorname{Mg}{ }_{I} \ldots \ldots .$. & 4057.52 & 4.34 & -1.20 & 1 & $\ldots$ & 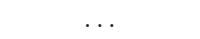 & $\ldots$ & $\ldots$ & $\ldots$ & $\ldots$ & $\ldots$ & \\
\hline $\operatorname{Mg}{ }_{1} \ldots \ldots . .$. & 4167.28 & 4.34 & -1.00 & 1 & 8.1 & 10.2 & 8.9 & 6.7 & 7.0 & $\ldots$ & $\ldots$ & 11.0 \\
\hline $\operatorname{Mg}$ I ......... & 4703.00 & 4.34 & -0.67 & 1 & 21.6 & 17.4 & 20.2 & 9.8 & 11.1 & $\ldots$ & $\ldots$ & $\ldots$ \\
\hline $\operatorname{Mg}_{I} \ldots \ldots$. & 5172.70 & 2.71 & -0.38 & 1 & 103.2 & 91.8 & 114.1 & 79.7 & 93.8 & 67.1 & 38.0 & 81.2 \\
\hline $\operatorname{Mg}_{I} \ldots \ldots .$. & 5183.62 & 2.72 & -0.16 & 1 & 119.5 & 102.5 & 134.3 & 85.3 & 97.8 & 69.9 & 51.2 & 83.8 \\
\hline Al I .......... & 3944.01 & 0.00 & -0.64 & 5 & 41.1 & 35.2 & $\ldots$ & 15.7 & 40.0 & 10.0 & $\ldots$ & $\ldots$ \\
\hline $\mathrm{Al}$ I .......... & 3961.52 & 0.00 & -0.34 & 5 & 43.2 & 40.8 & 46.8 & 26.6 & & 20.3 & 13.1 & 19.0 \\
\hline Si I.......... & 3905.53 & 1.91 & -1.04 & 3 & $\ldots$ & $\ldots$ & $\ldots$ & 51.0 & 92.8 & $\ldots$ & 26.9 & 57.5 \\
\hline
\end{tabular}

NotE.-Table 3 is presented in its entirety in the electronic edition of the Astronomical Journal. A portion is shown here for guidance regarding its form and content.

TABLE 4

Iron Abundances and Elemental Ratios for the Program Stars: Mg to Sc

\begin{tabular}{|c|c|c|c|c|c|c|c|c|c|c|c|c|c|c|c|c|c|c|c|c|c|}
\hline Star & $n$ & {$[\mathrm{Fe} \mathrm{I} / \mathrm{H}]$} & $\sigma$ & $n$ & {$[\mathrm{Fe} \mathrm{II} / \mathrm{H}]$} & $\sigma$ & $n$ & {$[\mathrm{Mg} / \mathrm{Fe}]$} & $\sigma$ & $n$ & {$[\mathrm{Al} / \mathrm{Fe}]$} & $\sigma$ & $n$ & {$[\mathrm{Si} / \mathrm{Fe}]$} & $\sigma$ & $n$ & {$[\mathrm{Ca} / \mathrm{Fe}]$} & $\sigma$ & $n$ & {$[\mathrm{Sc} / \mathrm{Fe}] \mathrm{II}$} & $\sigma$ \\
\hline HD $140283 \ldots$. & 49 & -2.43 & $(0.10)$ & 11 & -2.39 & $(0.13)$ & 5 & 0.32 & $(0.20)$ & 2 & -0.70 & $(0.09)$ & 1 & 0.14 & $\ldots$ & 7 & 0.27 & $(0.06)$ & 4 & 0.16 & $(0.11)$ \\
\hline $\mathrm{BD}+3^{\circ} 740 \ldots \ldots \ldots \ldots$ & 41 & -2.69 & $(0.11)$ & 8 & -2.63 & $(0.14)$ & 4 & 0.35 & $(0.16)$ & 2 & -0.64 & $(0.10)$ & 0 & $\ldots$ & $\ldots$ & 7 & 0.53 & $(0.05)$ & 3 & 0.27 & $(0.07)$ \\
\hline G139-8 .................. & 41 & -2.04 & $(0.11)$ & 11 & -2.10 & $(0.13)$ & 5 & -0.08 & $(0.21)$ & 2 & -0.99 & $(0.16)$ & 1 & -0.12 & $\ldots$ & 7 & 0.18 & $(0.07)$ & 3 & 0.46 & $(0.08)$ \\
\hline BS 17447-029 .......... & 12 & -2.91 & $(0.09)$ & 2 & -3.12 & $(0.03)$ & 2 & 0.02 & $(0.01)$ & 2 & -0.50 & $(0.18)$ & 0 & 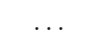 & $\ldots$ & 2 & 0.28 & $(0.04)$ & 1 & 0.25 & \\
\hline CS 22950-046 ........... & 42 & -3.27 & $(0.10)$ & 5 & -3.44 & $(0.14)$ & 5 & 0.44 & $(0.24)$ & 1 & -0.52 & $\ldots$ & 1 & 0.49 & $\ldots$ & 7 & 0.25 & $(0.06)$ & 4 & 0.23 & $(0.06)$ \\
\hline CS 22878-101 ........... & 44 & -3.07 & $(0.11)$ & 6 & -3.13 & $(0.09)$ & 5 & 0.68 & $(0.20)$ & 2 & -0.59 & $(0.06)$ & 1 & 0.39 & $\ldots$ & 7 & 0.32 & $(0.08)$ & 3 & 0.22 & $(0.11)$ \\
\hline HE $2133-1426 \ldots \ldots \ldots$ & 28 & -2.81 & $(0.10)$ & 3 & -2.88 & $(0.10)$ & 4 & 0.44 & $(0.12)$ & 2 & -0.46 & $(0.19)$ & 0 & $\ldots$ & $\ldots$ & 6 & 0.44 & $(0.12)$ & 3 & 0.38 & $(0.19)$ \\
\hline HE $2344-2800 \ldots \ldots \ldots$ & 44 & -2.53 & $(0.10)$ & 4 & -2.43 & $(0.08)$ & 4 & 0.20 & $(0.21)$ & 2 & -0.57 & $(0.13)$ & 0 & $\ldots$ & $\ldots$ & 5 & 0.29 & $(0.09)$ & 3 & 0.12 & $(0.18)$ \\
\hline HE $0024-2523 \ldots \ldots \ldots$ & 33 & -2.62 & $(0.19)$ & 2 & -2.57 & $(0.04)$ & 4 & 0.60 & $(0.14)$ & 1 & -0.38 & $\ldots$ & 0 & $\ldots$ & $\ldots$ & 3 & 0.59 & $(0.17)$ & 2 & 0.33 & $(0.13)$ \\
\hline HE $0130-2303 \ldots \ldots \ldots$ & 31 & -2.93 & $(0.11)$ & 2 & -2.96 & $(0.02)$ & 4 & 0.32 & $(0.23)$ & 2 & -0.60 & $(0.01)$ & 1 & -0.24 & $\ldots$ & 6 & 0.47 & $(0.11)$ & 2 & 0.35 & $(0.18)$ \\
\hline HE $0132-2429 \ldots \ldots \ldots$ & 31 & -3.56 & $(0.17)$ & 2 & -3.72 & $(0.06)$ & 4 & 0.38 & $(0.33)$ & 1 & -0.45 & $\ldots$ & 1 & 0.23 & $\ldots$ & 3 & 0.31 & $(0.13)$ & 3 & 1.18 & $(0.03)$ \\
\hline HE $0148-2611 \ldots \ldots . .$. & 26 & -2.96 & $(0.15)$ & 2 & -3.21 & $(0.09)$ & 2 & -0.02 & $(0.11)$ & 2 & -0.76 & $(0.06)$ & 0 & $\cdots$ & $\ldots$ & 3 & 0.32 & $(0.18)$ & 2 & 0.48 & $(0.20)$ \\
\hline HE $0218-2738 \ldots \ldots . .$. & 19 & -3.52 & $(0.14)$ & 0 & $\ldots$ & $\ldots$ & 2 & 0.06 & $(0.03)$ & 1 & -0.41 & $\ldots$ & 1 & -0.13 & $\ldots$ & 1 & 0.34 & $\ldots$ & 1 & 0.62 & $\ldots$ \\
\hline HE $0242-0732 \ldots \ldots .$. & 26 & -3.20 & $(0.19)$ & 4 & -3.36 & $(0.13)$ & 3 & 0.60 & $(0.32)$ & 1 & -0.65 & $\ldots$ & 1 & 0.13 & $\ldots$ & 5 & 0.46 & $(0.26)$ & 1 & 0.34 & \\
\hline
\end{tabular}



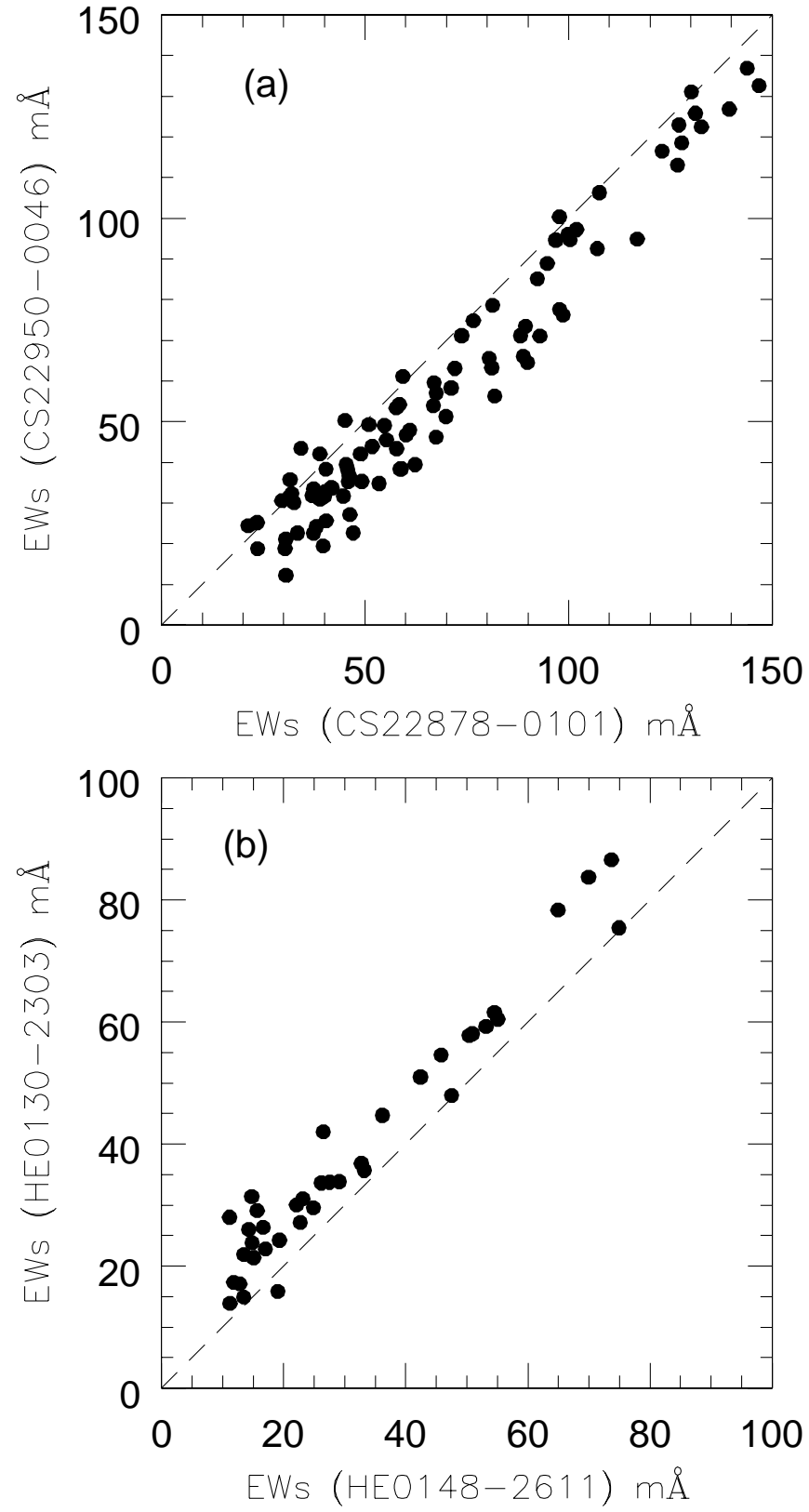

FIG. 2.-Comparison for the sets of EWs measured in $(a)$ a pair of giants and $(b)$ a pair of dwarfs of the present study, with similar atmospheric parameters.

total errors, we took into account the correlation between the error in $T_{\text {eff }}$ and the error in $\log g$ for each star that arises due to the procedure we adopt to derive surface gravities.

Internal errors in the microturbulent velocity can be checked by using the errors of the relationship between the abundances of $\mathrm{Fe} \mathrm{I}$ and the expected line strengths. Given the rather large number of measured $\mathrm{Fe}$ I lines and the wide range spanned by their intensities, the internal uncertainties in $v_{t}$ are quite small $\left( \pm 0.12 \mathrm{~km} \mathrm{~s}^{-1}\right)$, so that the $0.2 \mathrm{~km} \mathrm{~s}^{-1}$ adopted in Table 6 can be viewed as a conservative estimate. Unlike abundance analyses of solar-metallicity stars in this range of $T_{\text {eff }}$, in these very metal-poor stars the term arising from uncertainties in the microturbulent velocity $\left(v_{t}\right)$ does not dominate the abundance errors, because of the general overall weakness of the lines (save for a few isolated specific cases such as the $\mathrm{Mg} b$ and $\mathrm{Sr}$ II lines).
In the remaining part of this section, we discuss some relevant features of our analysis.

\subsection{Iron}

Iron abundances for our program stars are summarized in Table 4. There were no credible detections of any Fe II lines in the spectrum of one dwarf (HE 0218-2738) from the HES sample.

As we will see in the next subsection, the scatter in the differences between abundances derived from $\mathrm{Fe}$ I and by $\mathrm{Fe}$ II lines is quite small; we thus feel justified in assuming a constant offset between the Fe II and Fe I abundances of stars in our sample in order to obtain a value for the $[\mathrm{Fe}$ II $/ \mathrm{H}]$ ratio for the one star with no measured $\mathrm{Fe}$ II lines (HE 0218-2738). For this star only, we set Fe II $-\mathrm{Fe}_{\mathrm{I}}=-0.06$ dex. Abundance ratios of singly ionized species for this star given in Tables 4 and 5 are then referred to the abundance of Fe II obtained in this way.

According to the strict definition given in Paper I, six stars in the sample can be considered true EMP stars $([\mathrm{Fe} / \mathrm{H}]<-3$ dex $)$ : the two giants from the HK survey, and four dwarfs from the HES sample. Two other stars, BS 17447-029 and HE 0130-2303, are borderline, following this definition.

\subsubsection{Uncertainties in $\mathrm{Fe}$ Abundances}

Among the various diagnostics that can be used to test our Fe abundances, we considered the differences between the abundances derived from neutral and singly ionized $\mathrm{Fe}$ lines (hereafter $\Delta[\mathrm{Fe} / \mathrm{H}]_{\text {II-I }}$ ) and the slopes of the abundances derived from neutral Fe lines with respect to excitation potential $[\Delta(\mathrm{Fe} \mathrm{I} / \chi)]$. (See Figs. $4 a-4 b$, where both these quantities, derived for each star, are plotted against effective temperature. Values of these parameters are listed for each stars in the last two columns of Table 1.) These diagnostics are useful because both temperatures and gravities were derived independently of our line data (note however that we adjusted $v_{t}$ in order to reproduce similar abundances from weak and turbulence-sensitive lines). Ideally, we expect both $\Delta[\mathrm{Fe} / \mathrm{H}]_{\mathrm{II}-\mathrm{I}}$ and $\Delta(\mathrm{Fe} \mathrm{I} / \chi)$ to be null. However, there are various reasons why this might not occur in practice: (1) the atomic parameters adopted in our line analysis may themselves contain systematic offsets or trends; (2) our adopted effective temperature scale, or the theoretical isochrone used to derive gravities, may be incorrect; (3) the one-dimensional theoretical constant-flux model atmospheres used throughout this paper may be not an adequate representation of the real stellar atmospheres; (4) departures from LTE in the formation of Fe lines may significantly affect the derived abundances; and (5) observational errors both in the colors (affecting individual temperatures) and in the equivalent widths may introduce significant scatter. We leave aside here other possibilities, such as binarity of some stars, that might be used to explain individual discrepant points.

Clearly, the list of possible concerns is long. It is thus not surprising that both $\Delta[\mathrm{Fe} / \mathrm{H}]_{\mathrm{II}-\mathrm{I}}$ and $\Delta(\mathrm{Fe} \mathrm{I} / \chi)$ exhibit definite zero-point offsets and, also, possible trends with effective temperature (or, equivalently, luminosity). On average we have $\left\langle\Delta[\mathrm{Fe} / \mathrm{H}]_{\mathrm{II}-\mathrm{I}}\right\rangle=-0.07$ dex with $\sigma\left(\Delta[\mathrm{Fe} / \mathrm{H}]_{\mathrm{II}-\mathrm{I}}\right)=$ $0.11 \mathrm{dex}$ (in the sense that abundances from neutral Fe lines are larger than those obtained from singly ionized Fe lines; the error value is the rms scatter of the individual values for 


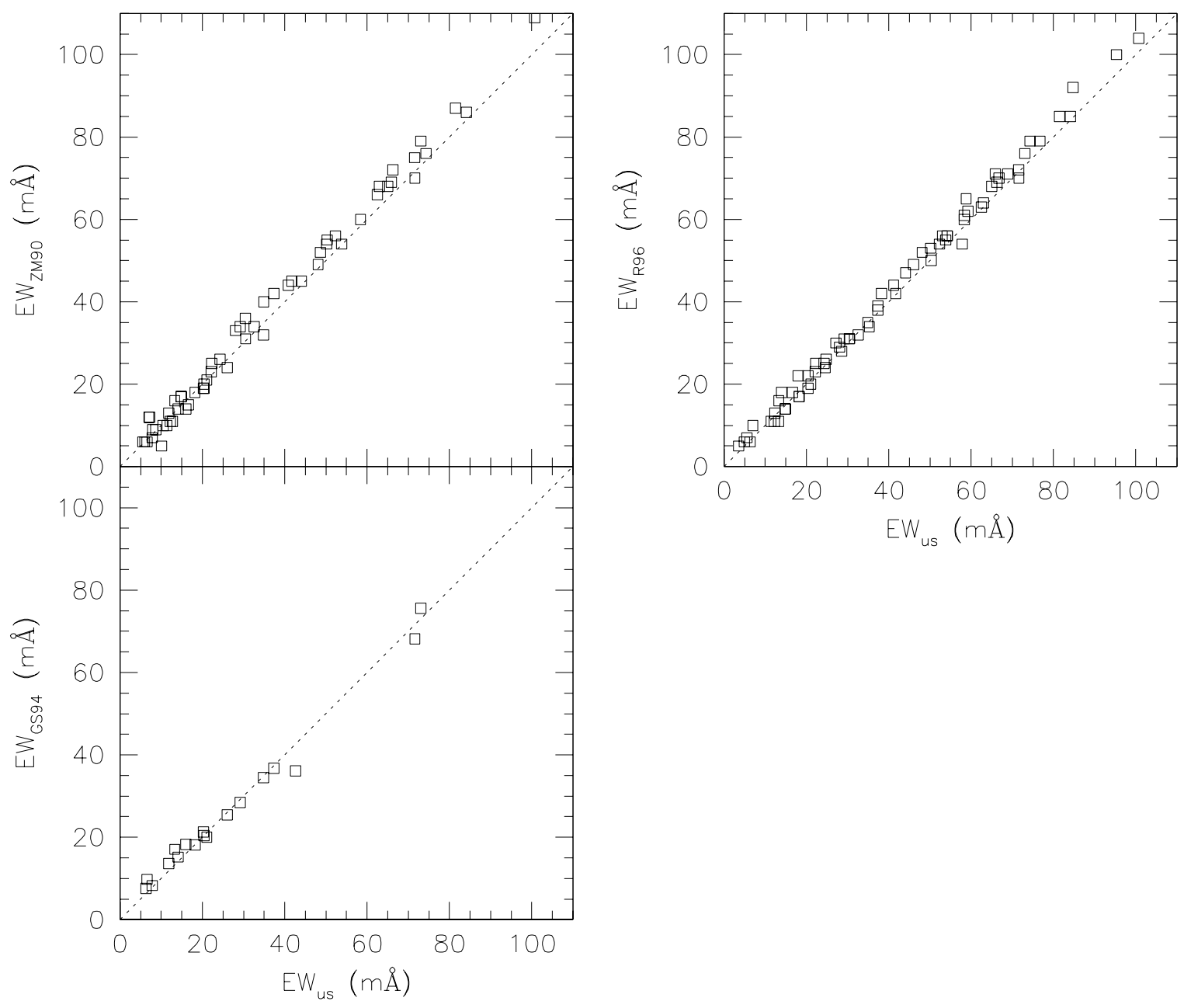

FIG. 3.-Comparison for the sets of EWs measured in HD 140283 in the present study with the works of Zhao \& Magain (1990, top left), Gratton \& Sneden (1994, bottom left), and Ryan et al. (1996, right). The dashed lines indicate equality.

each star) and $\langle\Delta(\mathrm{Fe} \mathrm{I} / \chi)\rangle=-0.046 \mathrm{dex}^{-1}$ with $\sigma[\Delta(\mathrm{Fe}$ $\mathrm{I} / \chi)]=0.025 \operatorname{dex} \mathrm{eV}^{-1}$ (where we have excluded $\mathrm{HE}$ 0132-2429 and HE 0242-0732, two stars that show obvious large trends of abundances with line excitation, which will be discussed below). If we exclude the two giants, the average value of $\Delta[\mathrm{Fe} / \mathrm{H}]_{\text {II-I }}$ becomes $-0.06 \pm 0.03$ dex, ${ }^{8}$ with a $\sigma=0.11$ dex (11 stars). To quantify these values in terms of uncertainties in the atmospheric parameters, we note that a difference in $\Delta(\mathrm{Fe} \mathrm{I} / \chi)$ of $0.05 \mathrm{dex} \mathrm{eV}^{-1}$ corresponds to a temperature difference of $\sim 250 \mathrm{~K}$, and that a difference of 0.07 dex in the iron abundances derived from $\mathrm{Fe} \mathrm{I}$ and $\mathrm{Fe}$ II implies a difference of about 0.16 dex in $\log g$.

When considering the implications of these tests, several points should be taken into account:

1. The Fe oscillator strengths we used throughout this paper are the best determinations that we found in the literature; they are discussed at length in a number of papers devoted to the solar Fe abundance (see, e.g., Asplund et al. 2000 and references therein). The transition probabilities for the Fe I lines were used as published, despite the fact that a small offset may exist between values obtained from the absorption experiments of the Oxford group and those from

${ }^{8}$ The difference is slightly larger for the two giants, being -0.11 dex. the selective laser-induced excitation experiments by the Hannover group. (A line-to-line comparison shows that these latter values are larger on average by $\sim 0.03$ dex.) Furthermore, the zero point of $\mathrm{Fe}$ II oscillator strengths is not yet firmly established, and possible offsets of several hundredths of a dex are easily possible (see the discussion in Asplund et al. 2000).

2. Even using the best available oscillator strengths, small offsets in the abundances are also present in a solar analysis done following precepts similar to those adopted for the program stars (LTE, one-dimensional model atmospheres from the Kurucz CD-ROMs, etc.) and EWs from Rutten \& van der Zalm (1984). In this case we find Fe abundances of $\log n(\mathrm{Fe})=7.512 \pm 0.012$ (with an rms scatter of 0.069 dex for individual lines) from $34 \mathrm{Fe}$ I lines, and $7.450 \pm 0.016$ (rms scatter of $0.085 \mathrm{dex}$ ) from $27 \mathrm{Fe}$ II lines (only lines with $\mathrm{EW}<100 \mathrm{m \AA}$ were considered, to reduce concerns related to the handling of collisional damping). ${ }^{9}$ In view of the roughness of the methods used, these abundances compare quite well with the much more sophisticated results obtained by most recent analyses of the solar photo-

\footnotetext{
${ }^{9}$ Throughout this paper, collisional damping was considered by multiplying the van der Waals broadening by an enhancement factor given by $\log E=\log (1+0.67 \mathrm{EP})$ (Simmons \& Blackwell 1982).
} 
TABLE 5

Elemental Ratios for the Program Stars: Ti to Ba

\begin{tabular}{|c|c|c|c|c|c|c|c|c|c|c|c|c|c|c|c|c|c|}
\hline Star & $n$ & {$[\mathrm{Ti} / \mathrm{Fe}] \mathrm{I}$} & $\sigma$ & $n$ & {$[\mathrm{Ti} / \mathrm{Fe}]$ II } & $\sigma$ & $n$ & {$[\mathrm{Cr} / \mathrm{Fe}]$} & $\sigma$ & $n$ & {$[\mathrm{Mn} / \mathrm{Fe}]$} & $\sigma$ & $n$ & {$[\mathrm{Sr} / \mathrm{Fe}] \mathrm{II}$} & $\sigma$ & $n$ & {$[\mathrm{Ba} / \mathrm{Fe}] \mathrm{II}$} \\
\hline $\mathrm{BD}+3^{\circ} 740 \ldots \ldots \ldots \ldots$ & 6 & 0.61 & $(0.04)$ & 16 & 0.39 & $(0.06)$ & 4 & -0.10 & $(0.08)$ & 3 & -0.48 & $(0.06)$ & 2 & -0.28 & $(0.02)$ & 1 & -0.71 \\
\hline BS $17447-029 \ldots \ldots \ldots$ & 0 & $\ldots$ & $\ldots$ & 11 & 0.34 & $(0.07)$ & 2 & -0.25 & $(0.03)$ & 2 & -0.59 & $(0.18)$ & 2 & 0.16 & $(0.06)$ & 1 & 0.41 \\
\hline CS 22950-046 .......... & 6 & 0.13 & $(0.06)$ & 17 & 0.18 & $(0.17)$ & 4 & -0.58 & $(0.12)$ & 3 & -0.93 & $(0.20)$ & 2 & -0.35 & $(0.04)$ & 1 & -1.37 \\
\hline CS 22878-101 ........... & 13 & 0.23 & $(0.10)$ & 20 & 0.24 & $(0.17)$ & 9 & -0.32 & $(0.24)$ & 3 & -1.05 & $(0.16)$ & 2 & -0.39 & $(0.13)$ & 1 & -1.08 \\
\hline HE $2133-1426 \ldots \ldots \ldots$ & 2 & 0.54 & $(0.07)$ & 11 & 0.27 & $(0.11)$ & 2 & -0.16 & $(0.05)$ & 1 & -0.41 & & 2 & -0.25 & $(0.05)$ & 1 & -0.60 \\
\hline HE $0130-2303 \ldots \ldots . .$. & 0 & $\ldots$ & 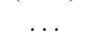 & 13 & 0.46 & $(0.13)$ & 3 & -0.10 & $(0.14)$ & 1 & -0.43 & & 2 & 0.06 & $(0.09)$ & 1 & -0.91 \\
\hline HE $0132-2429 . . \ldots \ldots .$. & 5 & 0.51 & $(0.06)$ & 15 & 0.64 & $(0.08)$ & 3 & -0.51 & $(0.05)$ & 2 & -0.87 & $(0.03)$ & 2 & 0.81 & $(0.12)$ & 1 & -0.98 \\
\hline HE $0148-2611 \ldots \ldots . .$. & 0 & $\ldots$ & $\ldots$ & 9 & 0.37 & $(0.14)$ & 2 & -0.29 & $(0.08)$ & 0 & $\cdots$ & $\ldots$ & 2 & -0.82 & $(0.12)$ & 0 & $\ldots$ \\
\hline HE $0218-2738 \ldots \ldots . .$. & 0 & $\ldots$ & $\ldots$ & 9 & 0.62 & $(0.12)$ & 2 & -0.16 & $(0.14)$ & 0 & $\cdots$ & & 1 & -0.43 & 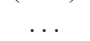 & 0 & $\cdots$ \\
\hline HE $0242-0732 \ldots \ldots \ldots$ & 0 & $\ldots$ & $\ldots$ & 3 & 0.25 & $(0.05)$ & 2 & -0.37 & $(0.14)$ & 0 & $\ldots$ & $\ldots$ & 1 & -1.23 & $\ldots$ & 0 & $\ldots$ \\
\hline
\end{tabular}

spheric spectrum (e.g., Asplund et al. 2000). Our solar analysis yields $\langle\Delta(\mathrm{Fe} \mathrm{I} / \chi)\rangle=-0.023 \mathrm{dex} \mathrm{eV}^{-1}$ with $\sigma[\Delta(\mathrm{Fe} \mathrm{I} /$ $\chi)]=0.009 \mathrm{dex} \mathrm{eV}^{-1}$, which is quite similar to the value we obtain for our very metal-poor program stars. Note, however, that the set of lines used in this solar analysis is disjoint from that used for the stellar analyses, because those lines measurable in extremely metal-poor stars are heavily saturated in the solar spectrum. Line selection is of particular importance for the determination of $\Delta(\mathrm{Fe} \mathrm{I} / \chi)$, which is also sensitive to the value adopted for the microturbulent velocity. On the other hand, trends of abundances with line excitation have also been found in previous, much more accurate analyses of the solar photospheric spectrum (see, e.g., Grevesse \& Sauval 1999). In this respect, it is interesting to note that such a trend is not present when three-dimensional models are used to analyze Fe lines in the solar spectrum (Asplund et al. 2000). Three-dimensional effects may be expected to have an even larger impact on the analysis of metal-poor stars than in the case of the Sun (see Asplund et al. 1999). In fact, because of the lower opacity in the atmospheres, the lines are expected to form deeper in the stars, where the impact of convection is larger and, possibly, the granulation contrast is larger as well.

3. The rms scatter of the individual values of $\Delta[\mathrm{Fe} / \mathrm{H}]_{\mathrm{II}-\mathrm{I}}$ $(0.11 \mathrm{dex})$ roughly agrees with the expected uncertainties. To show this, we assumed that the internal errors in $T_{\text {eff }}$ are those estimated in Paper I, that is, $\pm 75 \mathrm{~K}$, possibly increasing up to $\pm 150 \mathrm{~K}$ for turnoff stars only. To estimate the corresponding errors in $\log g$, one needs the slope of the isochrone in the region of interest, namely, $\Delta[\log (g) /$ $T_{\text {eff }} \sim 0.003$ dex $\mathrm{K}^{-1}$ along the red giant branch (RGB), and much less $\left(\sim 0.0005\right.$ dex $\left.\mathrm{K}^{-1}\right)$ for less evolved evolutionary stages (subgiants and dwarfs). Below the main-sequence turnoff, the slope reverses in sign. Reading the changes in $\mathrm{Fe} \mathrm{I}$ and $\mathrm{Fe}$ II abundances expected for a $100 \mathrm{~K}$ increase in $T_{\text {eff }}$ from Table 6 (with consequent changes in $\log g$ and overall metallicity $[\mathrm{A} / \mathrm{H}])$, the resulting value of $\Delta[\mathrm{Fe} / \mathrm{H}]_{\mathrm{II}-\mathrm{I}}$ would be about +0.08 dex for giants and -0.04 and -0.08 dex for subgiants and dwarfs, respectively. When we sum these values in quadrature with the internal error due to uncertainties in the measured EWs, for our Fe II abundances (typically 0.05 dex for giants and 0.09 dex for dwarfs, where

TABLE 6

Sensitivity of Abundances to Atmospheric Parameters

\begin{tabular}{|c|c|c|c|c|c|c|c|c|c|c|}
\hline RATIO & \multicolumn{5}{|c|}{ CS 22950-046 } & \multicolumn{5}{|c|}{ HE $2344-2800$} \\
\hline$[\mathrm{Mg} / \mathrm{Fe}] \ldots \ldots \ldots$ & -0.039 & 0.010 & 0.005 & 0.036 & 0.044 & -0.017 & -0.010 & 0.000 & -0.002 & 0.020 \\
\hline$[\mathrm{Al} / \mathrm{Fe}] \ldots \ldots \ldots$ & -0.031 & -0.004 & -0.005 & -0.049 & 0.062 & -0.004 & 0.004 & 0.000 & 0.019 & 0.019 \\
\hline$[\mathrm{Si} / \mathrm{Fe}] \ldots \ldots \ldots . .$. & 0.004 & -0.044 & -0.008 & -0.002 & 0.063 & 0.000 & 0.007 & 0.000 & 0.011 & 0.011 \\
\hline$[\mathrm{Ti} / \mathrm{Fe}]$ I .......... & 0.012 & 0.032 & 0.011 & 0.058 & 0.084 & 0.006 & 0.004 & 0.001 & 0.027 & 0.028 \\
\hline$[\mathrm{Ti} / \mathrm{Fe}]$ II ........ & 0.037 & -0.004 & 0.002 & -0.021 & 0.037 & 0.021 & -0.003 & 0.000 & -0.003 & 0.020 \\
\hline$[\mathrm{Cr} / \mathrm{Fe}] \mathrm{I} \ldots \ldots .$. & -0.001 & 0.023 & 0.004 & 0.017 & 0.038 & 0.007 & 0.004 & 0.001 & 0.014 & 0.016 \\
\hline$[\mathrm{Mn} / \mathrm{Fe}] \ldots \ldots \ldots$ & 0.012 & 0.012 & -0.004 & -0.047 & 0.056 & 0.021 & 0.004 & 0.003 & 0.009 & 0.024 \\
\hline$[\mathrm{Fe} \mathrm{I} / \mathrm{H}] \ldots \ldots \ldots$ & 0.103 & -0.043 & -0.017 & -0.066 & 0.078 & 0.072 & -0.007 & 0.003 & -0.030 & 0.076 \\
\hline$[\mathrm{Fe}$ II $/ \mathrm{H}] \ldots \ldots$. & 0.020 & 0.051 & 0.008 & -0.046 & 0.107 & 0.017 & 0.069 & 0.002 & -0.019 & 0.039 \\
\hline
\end{tabular}



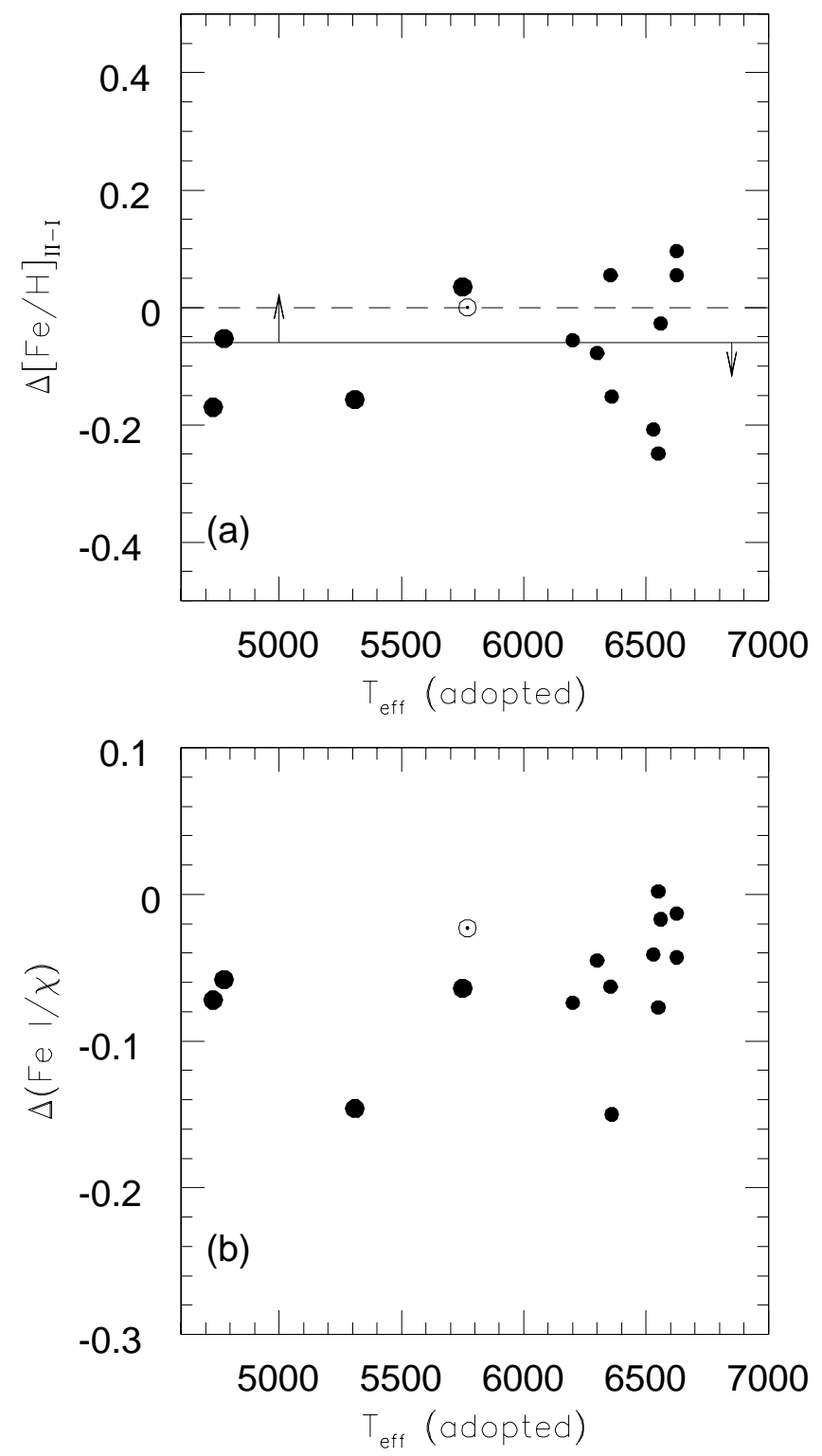

FIG. 4.-(a) Differences of abundances of iron from singly ionized and neutral lines as a function of adopted temperatures for program stars. The dashed line is the value for the Sun, while the solid line is the average value from dwarfs in our sample. Arrows on the left and on the right side of the figure indicate the change in $\mathrm{Fe}$ II $-\mathrm{Fe}$ I resulting from a change of $+100 \mathrm{~K}$ in $T_{\text {eff }}$ for a giant and a dwarf, respectively. (b) Slope of the relationships of iron abundances $\log n(\mathrm{Fe} \mathrm{I})$ vs. excitation potential as a function of the adopted $T_{\text {eff. }}$ The circled dot is the position of the Sun in these plots. In both panels (and in following figures) larger symbols indicate giant and/or subgiant stars in our sample, i.e., stars with surface gravity greater than $\log g=3.8$

only a few lines were usually observed), we find that the observed scatter in the values of $\Delta[\mathrm{Fe} / \mathrm{H}]_{\text {II-I }}$ is reproduced.

4. An interesting feature of our analysis is that adoption of a systematically incorrect $T_{\text {eff }}$ scale would, as described above, yield values of $\Delta[\mathrm{Fe} / \mathrm{H}]_{\text {II-I }}$ of opposite signs for dwarfs and giants when the resulting changes in $\log g$ were included. However, a larger sample is probably needed to explore this possibility. Again, adoption of realistic threedimensional model atmospheres for the program stars might provide better insight into this issue (Asplund et al. 1999).
For most of the sample stars, the general trends shown in Figure 4 can probably be explained by $T_{\text {eff }}$ scale errors or model atmosphere uncertainties. However, two stars (HE 0132-2429 and HE 0242-0732) display trends of abundance with excitation potential $\left[\Delta\left(\mathrm{Fe}_{\mathrm{I}} / \chi\right) \leq-0.15\right]$ that are much larger than can be induced by typical errors in the stellar parameters, primarily in the effective temperature. The trend found for HE 0132-2429 is clearly significant and does not depend on inclusion or not of one or two lines (see Fig. 5). The situation for HE 0242-0732 was less clear, since most of the apparent trend obtained when the atmospheric parameters of Paper I were used for this star was due to a single line.

In order to remove these trends, effective temperatures much lower (by $\sim 500 \mathrm{~K}$ ) than those given by the colors would be required. Such differences cannot be due to the reddening corrections, because these are too small. Furthermore, effective temperatures derived from the $\mathrm{H} \delta$ profiles support the high $T_{\text {eff }}$ values given by the colors (see Paper I;

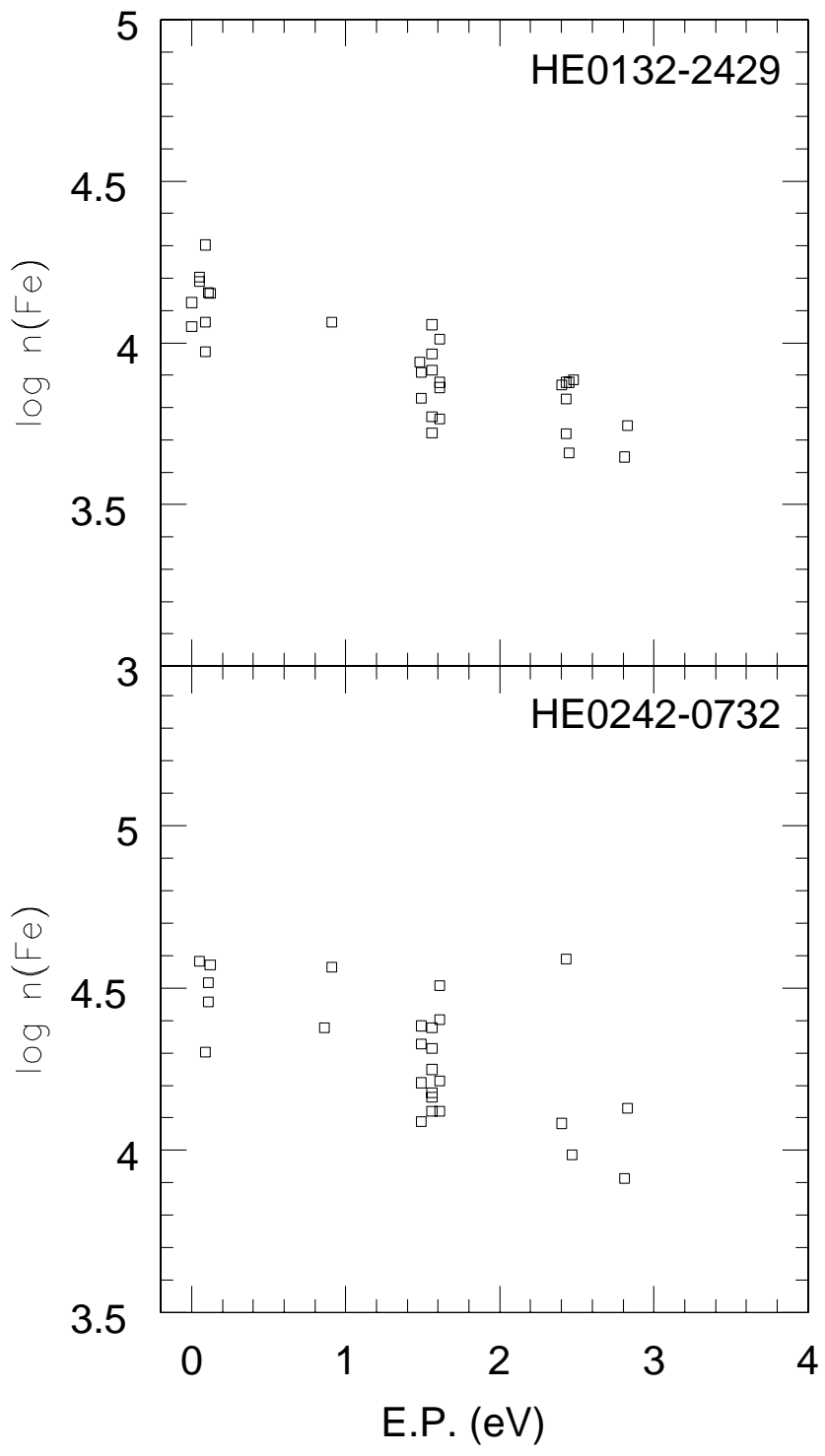

FIG. 5.-Run of iron abundances with excitation potential for HE 0132-2429 and HE 0242-0732, the two stars that appear anomalously low in Fig. 4. 
however, we note that $\mathrm{H} \delta$ is quite weak for $\mathrm{HE}$ 0132-2429, so a precise determination of $T_{\text {eff }}$ is not easy for this star). Also, it does not seem possible to invoke contamination by a bluer companion such as a main-sequence turnoff star (this explanation may obviously only be considered for $\mathrm{HE}$ 0132-2429). To demonstrate this, we note that the observed (dereddened) $V-K$ color for this star is $V-K=1.86$. This color can be obtained by combining the flux from a star along the RGB (with $M_{V}=1.4$ and $V-K=1.91$ ) and a star at the main-sequence turnoff $\left(M_{V}=3.8, V-K=1.05\right)$. In the (observed) blue part of the spectrum, the secondary would be about 7 times less luminous than the primary: it would be difficult to detect such a star if the radial velocity difference were small. However, the difference between the color of the primary and that of the whole system would be only $0.05 \mathrm{mag}$ in $V-K$, corresponding to less than $100 \mathrm{~K}$ in $T_{\text {eff. }}$ This difference is far too small to explain the trend of Figure 5.

In the case of HE 0242-0732, the automatic EW-measuring routine did not pick up any Fe II lines. Hence we measured the EWs of the four strongest Fe II lines expected to be present in our spectra, at 4233.16, 4923.93, 5018.45, and $5169.03 \AA$, by hand. All these lines are only a bit stronger than noise, so their EWs are somewhat uncertain; however, they surely are not much stronger than our estimates. Using these lines, a lower limit for the surface gravity may be obtained by assuming that $\log n(\mathrm{Fe} \mathrm{I}) \sim \log n(\mathrm{Fe}$ II $)$. However, the Fe II lines in this star are clearly much weaker than expected on the basis of the atmospheric parameters used thus far $\left(T_{\text {eff }}=6455\right.$ and $\left.\log g=4.2\right)$ from Paper I. This forced us to consider both a lower temperature and a less evolved evolutionary phase for this star. A lower temperature is also suggested by the $\mathrm{H} \delta$ profile.

In Paper I, we already noted that the $K$ magnitude for this star from the 2MASS is quite uncertain (it is the faintest star in the infrared in our sample). On the whole, for this star it

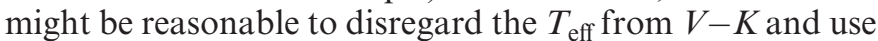
only the temperature from the more accurate $V-J$ color, that is, to adopt a $T_{\text {eff }}$ of $6360 \mathrm{~K}$. Also, it may be reasonable to consider a gravity consistent with a main-sequence star at this $T_{\text {eff }}(\log g=4.4)$, rather than a star brighter than the turnoff.

We then repeated the analysis with the revised stellar parameters, after adding manual measurements of four more weak $\mathrm{Fe}$ I lines, to extend the range of excitation potential (EP) sampled. With the same procedure adopted above, we derive a full set of parameters for HE 0242-0732 of $\left(T_{\text {eff, }}, \log g,[\mathrm{Fe} / \mathrm{H}], v_{t}\right)=(6360,4.4,-3.21,0.40)$. The abundance from Fe II lines is still lower than that obtained from $\mathrm{Fe}_{\mathrm{I}}$ lines, although the difference is roughly halved with these parameters with respect to the original discrepancy. We regard this new analysis as quite robust, and we adopt these new parameters for this star.

Using these new parameters and the slightly more extended line list, the discrepancy of star HE 0242-0732 in Figure 4 is reduced. There is still a trend of abundances with $\mathrm{EP}$, but it is well within the error bars, which are large, as a consequence of the small range in EP of available Fe I lines. The trend is not very different from the trends shown by the other stars in the sample. Another improvement achieved using this set of atmospheric parameters is that the Ti II abundance (which was previously the lowest in the sample) is now raised to +0.25 dex and does not stand out anymore as an outlier in Figure 7 (see below).

\subsubsection{Comparison with Results from a Purely Spectroscopic Analysis}

As a further check of our adopted procedure to derive atmospheric parameters, we performed a purely spectroscopic analysis, deriving temperatures, surface gravities, overall metal abundance, and microturbulent velocities from the equivalent widths alone. In this process, we ignored the photometry that forms the basis of the assignment of stellar parameters used in Paper I.

We adopted HE 0242-0732 as typical of our program stars (it is neither the best nor the worst case, as far as the quality of observational material is concerned; however, it does represent an extreme case of differences between results of our original analysis [with temperatures based on colors, and gravities from the color-magnitude diagram] and those from a purely spectroscopic analysis). The spectroscopic set of parameters in this case is an effective temperature $T_{\text {eff }}=5250 \mathrm{~K}$ (obtained by zeroing the slope in the relation of $\mathrm{Fe}$ abundances with the EP of lines), a surface gravity $\log g=2.55$ (assuming that $\mathrm{Fe}$ I and Fe II lines must yield the same iron abundance), $[\mathrm{A} / \mathrm{H}]=-4.21$ dex (given by the abundance of iron), and a microturbulent velocity of 0.45 $\mathrm{km} \mathrm{s}^{-1}$ (derived by eliminating trends of abundances with expected line strength for Fe I lines).

This set of parameters is quite different from that of our original analysis. To estimate the appropriate error bars, we must take into account the covariance among the errors, and hence we performed the following exercise: Using this set as a starting point, we used the same set of Fe lines to repeat the analysis, changing the $v_{t}$ value until the $1 \sigma$ value from the slope of the abundance versus line-strength relation was reached and at the same time optimizing the other parameters at their best value. The resulting set $\left(T_{\text {eff }}, \log g\right.$, $\left.[\mathrm{A} / \mathrm{H}], v_{t}\right)$ was $(5480,2.85,-4.07,0.74)$. Starting then from this second set of parameters, we changed the effective temperature in order to have all parameters optimized when the $1 \sigma$ value from the starting slope of the abundance versus EP relation was reached (still leaving the trend of abundances with expected line strength off by $1 \sigma$ from its best value). In this way we obtain a third set of parameters: (5760, 3.45, $-3.81,0.78)$. Finally, we repeated the same exercise, but now achieving a change of $1 \sigma$ in the difference between Fe I and Fe II (again, leaving trends of abundances with expected line strength and excitation off from their best value by $1 \sigma$ ). The resulting final set of parameters was $(5780,3.05,-3.79$, $0.88)$.

We emphasize here that this last set of parameters is still statistically acceptable: each of the residual trends is not larger than its $1 \sigma$ rms error. A simple comparison between this last set and our starting point allows us to give an estimate of the $1 \sigma$ internal errors associated with a purely spectroscopic analysis; we consider these values more appropriate than those derived from considering each individual parameter as independent from the others. Uncertainties are $\pm 530 \mathrm{~K}$ in $T_{\text {eff }}, \pm 0.43 \mathrm{~km} \mathrm{~s}^{-1}$ in $v_{t}$, and $\pm 1.3 \mathrm{dex}$ in $\log g$. On the whole, the uncertainty in the overall metal abundance from the purely spectroscopic analysis is \pm 0.42 dex! This very large error bar is more than twice that deduced by assuming that the atmospheric parameters are independent of each other. This is mainly due to the correlation existing between EP and line strength for Fe I lines. We conclude that the intrinsic uncertainties in a purely spectroscopic approach are too large to secure a robust result when 
dealing with extremely metal-poor stars, for which the number of reliable lines measured is rather small, particularly for Fe II, uncertainties in the EWs of individual lines are not negligible, and the range in excitation potential is limited.

For these reasons, we think that the procedure we have adopted, which fixes temperature and gravity by independent means, works better in our case. The previous exercise, for example, shows that by using only information provided by Fe lines (as is done in the purely spectroscopic analysis), we could not say whether HE $0242-0732$ is an RGB or horizontal-branch star $(5250 \mathrm{~K}, 2.55 \mathrm{dex})$, or a subgiant (5780 $\mathrm{K}, 3.05 \mathrm{dex})$. Note that both these combinations of $T_{\mathrm{eff}}$ and $\log g$ are compatible with the same isochrone and would thus appear to be plausible solutions.

\subsubsection{Checks on Departures from LTE for $\mathrm{Fe}$}

Having derived gravities from $T_{\text {eff }}$ and isochrones only, we can in principle use the ionization balance of Fe in order to check whether Fe abundances are affected by departures from LTE. This issue is the subject of considerable debate, since Thévenin \& Idiart (1999) suggested that LTE analyses tend to underestimate the Fe I abundances (by a few tenths of a dex in extremely metal-poor stars), while Gratton et al. (1999) pointed out that large ( $>0.2$ dex) departures from LTE are unlikely, and LTE should instead be a very good assumption for Fe line formation in metal-poor dwarfs. In view of the large uncertainties still present in the collisional cross sections (see, e.g., Gehren et al. 2001), and given the limitations in the adopted model atoms, there is no way at present to decide from first principles which of these analyses is valid.

There is no evidence in our data of a large Fe overionization, judging from Figure $4 a$. As discussed above, the average difference in abundances from $\mathrm{Fe}$ I and $\mathrm{Fe}$ II lines is only marginally different from zero. Moreover, almost all stars show differences that have the wrong sign for overionization. This seems to rule out large departures from LTE in the formation of $\mathrm{Fe}$ lines in metal-poor stars.

On the other hand, the discussion in $\S 4.1 .1$ showed that there are so many possible uncertainties still remaining in the temperature scale, in the model atmospheres, and in the oscillator strengths that small non-LTE effects (at a level of $0.1-0.2$ dex) cannot be firmly excluded from our data.

\subsection{The $\alpha$-Elements}

We detected lines arising from two stages of ionization for titanium, and hence we can use the ionization equilibrium of this element as an additional clue for the presence of non-LTE effects. In fact, given the lower ionization potential of Ti I, it may be expected that this element ought to be even more vulnerable to non-LTE effects than is iron. There is no conclusive evidence for departure from LTE from the Ti I/Ti II abundance. From the data in Table 5, a trend of decreasing Ti II $-\mathrm{Ti}$ I differences as $T_{\text {eff }}$ increases seems to be present, with the two giants showing somewhat larger (positive, i.e., abundances from Ti II larger than those from neutral Ti) differences. However, even in this case we believe that the safest approach is not to draw any firm conclusions until a larger sample becomes available. We are concerned that there is a possible bias resulting from the rather uncertain $\mathrm{Ti}$ I abundances in the warm, metal-poor dwarfs that are clustered around $\mathrm{Ti}$ II $-\mathrm{Ti}$ I $\sim-0.2$ to -0.3 dex. Moreover, we are not aware of any computations of non-LTE effects for titanium.

We note in passing that data from McW95, and the few stars from RNB96 with both Ti I and Ti II measured (all giant or subgiant stars), show the same pattern of differences in titanium abundances as a function of metallicity, with an average value of $\mathrm{Ti}$ II - Ti I just below zero; values as low as about -0.3 dex can be found.

\subsection{Aluminum}

For aluminum, the only accessible feature in our HIRES spectra is the resonance doublet at 3944-3961 A. However, a reliable measurement of these lines is somewhat hampered by the proximity of $\mathrm{H} \epsilon$ to the $3961 \AA$ line. Moreover, the line at $3944 \AA$ is disturbed by $\mathrm{CH}$ features in some of our stars (Arpigny \& Magain 1983).

Apart from these problems, this doublet is not an ideal abundance indicator in view of the presence of possible large departures from LTE, extensively discussed by Baumüller \& Gehren (1997). They found that LTE analyses produce a large underestimate of $\mathrm{Al}$ abundances; they give nonLTE corrections (about $0.6 \mathrm{dex}$ ) for the resonance lines in their Table 1.

Very recently, Gratton et al. (2001) found that inclusion of these corrections improves the agreement between $\mathrm{Al}$ abundances derived in globular cluster dwarfs from this doublet and from the high-excitation infrared doublet at 8772.9-8773.9 $\AA$, which is believed to be less affected by departures from LTE. This provides further support for the calculations of Baumüller \& Gehren. We therefore interpolated Table 1 of Baumüller \& Gehren (1997) to derive corrections to the $\mathrm{Al}$ abundances listed in Table 4.

\subsection{The Iron-Peak Elements}

Apart from Fe, our spectra provide useful information on the abundances of three additional Fe-peak elements (Sc, $\mathrm{Cr}$, and $\mathrm{Mn}$ ). Lines of the remaining elements are too weak to be reliably measured, because of the combination of low metal abundance and high temperature for most of our stars.

Lines of Sc and Mn exhibit hyperfine structure (HFS), mainly due to their nonzero nuclear magnetic moments. HFS is expected to be rather narrow for the Sc II lines, with small impact on abundances, and hence we neglect it. On the other hand, Mn lines exhibit broad HFS; this was taken into account using data from Booth, Shallis, \& Wells (1983).

\subsection{The Neutron-Capture Elements}

We measured lines for two $n$-capture elements ( $\mathrm{Sr}$ and $\mathrm{Ba}$; in both cases, we observed the resonance lines of the singly ionized species). The Eu II line at $4129.7 \AA$ is within the observed spectral region, but it is too weak to be detectable in any of our spectra. ${ }^{10}$ Note that none of our stars belong to the group of rare stars that exhibit strong overabundances of elements produced by the $r$-process, which includes CS 22892-052 and CS 31082-001. The classification of BS

\footnotetext{
${ }^{10}$ For an EMP dwarf near the main-sequence turnoff with $[\mathrm{Fe} / \mathrm{H}]=-3.0 \mathrm{dex}$, an upper limit of $10 \mathrm{m \AA}$ for the EW of the strongest $\mathrm{Eu}$ II line corresponds to $[\mathrm{Eu} / \mathrm{Fe}] \lesssim+1.8$ dex, too large to provide any interesting constraints.
} 
17447-029 is somewhat uncertain; this star shows high abundances of $\mathrm{Sr}$ and $\mathrm{Ba}$ but does not have detected $\mathrm{Eu}$ (or any strong $\mathrm{CH}$ features). This star may be mildly $r$-process enhanced.

HFS due to both nonzero magnetic moment and isotopic splitting is significant for the Ba II line at $4554.04 \AA$. The HFS corrections were evaluated using the data of Steffen (1985); those recently calculated by McWilliam (1998) are very similar. Note, however, that we do not know the relative abundances of the different $\mathrm{Ba}$ isotopes: our assumed distribution is the solar one and may be inappropriate for metal-poor stars.

\section{DISCUSSION}

\subsection{Results from the Keck Pilot Program}

\subsubsection{Comparison for Stars in Common with McWilliam et al.}

There are two stars in common between our sample and that analyzed by McW95: the two giants CS 22878-101 and CS 22950-046. The adopted $T_{\text {eff }}$ values are quite similar (ours being larger on average by $38 \mathrm{~K}$ ); we adopted higher surface gravities (on average by $0.3 \mathrm{dex}$ ) and smaller microturbulent velocities (on average by $0.52 \mathrm{~km} \mathrm{~s}^{-1}$ ). The effects of these differing choices of $\log g$ and $v_{t}$ are in most cases similar in magnitude but of opposite sign and so somewhat cancel out. The differences between our and the McW95 analysis are as follows (in the sense ours minus McW95; in parentheses we give the difference expected from the atmospheric parameters): $\mathrm{Fe},+0.09$ (0.13); $\mathrm{Mg},+0.15$ (-0.09); $\mathrm{Al},-0.17$ (0.11); Si, +0.04 (-0.07); Ca, -0.15 (-0.09); Sc, +0.17 (0.07); Ti I, -0.24 (-0.09); Ti II, -0.04 (0.06); $\mathrm{Cr}$, +0.02 (0.01); Mn, -0.19 (0.14); Sr, +0.20 (0.24); Ba, -0.30 (0.02).

In several cases, there is a reasonable agreement between the observed and expected offsets. The large difference for $\mathrm{Mg}$ is due to the $g f$-values that we adopted (see the Appendix). The differences for $\mathrm{Al}$ and $\mathrm{Si}$ are discussed elsewhere in the text. Part of the difference for $\mathrm{Sc}$ is due to the fact that we neglected the HFS for this element (while it is considered by McW95).

\subsection{2. $\alpha$-Elements}

Abundances for the $\alpha$-elements are summarized in Tables 4 and 5 and shown in the left panels of Figures 6 and 7. We also show, for purpose of comparison, the results, from the literature, of previous high-dispersion studies: McW95 for a sample of 33 metal-poor giants, and a compilation of literature data taken from NRB01. In addition to the five EMP giants they studied, NRB01 used high-quality data compiled from the literature to discuss the chemical evolution of the Galaxy. In the construction of Figures 5-9, 11, and 12 and of Table 7, we employ three data sets from that paper: (1) the data from the Ryan-Norris-Beers group (Ryan, Norris, \& Bessell 1991; RNB96; Norris, Beers, \& Ryan 2000; NRB01; hereafter collectively RNB), (2) the data from Gratton and Sneden (Gratton 1989; Gratton \& Sneden 1987, 1988, 1991, 1994), and (3) the data from Stephens (1999).

Following Table 1 in NRB01, the data from McW95 come from lower quality spectra, but $\alpha$-elements have strong lines in metal-poor stars, and hence concerns due to somewhat lower resolution and $\mathrm{S} / \mathrm{N}$ are limited in this case. The overall patterns for each of the $\alpha$-elements are quite similar, even though our sample is mostly composed of dwarfs. Unweighted mean values for $[\mathrm{Mg} / \mathrm{Fe}],[\mathrm{Ca} / \mathrm{Fe}]$, $[\mathrm{Ti} / \mathrm{Fe}] \mathrm{I}$, and $[\mathrm{Ti} / \mathrm{Fe}] \mathrm{II}$ are respectively $0.31(\sigma=0.24),{ }^{11}$ $0.35(\sigma=0.12), 0.40(\sigma=0.19)$, and $0.36(\sigma=0.15)$ dex, to be compared with $0.40(\sigma=0.22), 0.44(\sigma=0.13), 0.32$ $(\sigma=0.19)$, and $0.30(\sigma=0.13)$ in the McW95 sample. The average difference in the sense $[\alpha / \mathrm{Fe}]_{\text {us }}-[\alpha / \mathrm{Fe}]_{\text {McW95 }}$ is only $-0.01, \sigma=0.08$ dex, suggesting that there is no significant offset in the mean $\alpha$-element abundances between metal-poor dwarfs and giants.

The only apparent offset seems to lie in the Si abundances. However, our $[\mathrm{Si} / \mathrm{Fe}]$ values are based on only one strong line, Si I $\lambda 3905$, and this line was disregarded by McW95 because it exceeded their adopted reduced equivalent width limit. Unfortunately, all other Si lines in the spectral region sampled by our spectra are vanishingly weak in these warm, metal-poor stars. The large scatter in the plot of $[\mathrm{Si} / \mathrm{Fe}]$ versus $[\mathrm{Fe} / \mathrm{H}]$ is present in all samples of metal-poor stars, but the observational problems suggest that this could be an artifact of the still uncertain abundance analysis for this ion.

We note that four out of 14 stars in our sample exhibit distinctly low $[\mathrm{Mg} / \mathrm{Fe}]$ ratios, close to the solar ratio. It would be interesting to have more insight into these objects by coupling chemical data with kinematic information. This is deemed important, since more and more stars that show underabundances among the $\alpha$-elements are being discovered by several investigators, for example, stars with $[\mathrm{Fe} / \mathrm{H}]<-2$ dex and $[\mathrm{Mg} / \mathrm{Fe}]<0.1 \mathrm{dex}$ in the top left panel of Figure 6. All our low-magnesium stars are dwarfs, but they are equally intermingled with giants in other samples. Often, when orbital parameters can be estimated, these objects are seen to have large apogalacticon distances, suggesting their possible origin in lower mass stellar systems with chemical evolution histories that were distinctly different from that of our Galaxy and which were subsequently accreted into our Galaxy (see King 1997; Nissen \& Schuster 1997; Carney et al. 1997).

At present we do not have good information about Galactic orbits for all the objects in our sample, but the expected extension of the US Naval Observatory CCD Astrograph Catalog (Zacharias et al. 2000) from the southern sky to the northern sky, as well as the forthcoming release of proper motions from the Guide Star Catalog II (and other ongoing proper-motion programs) should fill the six-dimensional parameter space, when coupled to the radial velocity information. We thus defer the study of possible correlations of chemistry and kinematics to future work.

The scatter seen in $\mathrm{Ca}$ abundances is similar to that in the McW95 stars, if one disregards the star with $[\mathrm{Ca} / \mathrm{Fe}] \sim 0.9$ in their sample, and it seems to be lower than the scatter observed in the RNB data. Our elemental ratios $[\mathrm{Ca} / \mathrm{Fe}]$ seem to be slightly lower, on average, than those from McW95, and more in agreement with data from RNB, even if we do not find stars having very low $\mathrm{Ca}$ abundances $([\mathrm{Ca} / \mathrm{Fe}]<0.1)$. Excluding the four most metal-poor stars in the McW95 sample, the other data sets do not show any trend of $\mathrm{Ca}$ abundances increasing for decreasing metal abundances.

11 Throughout this paper, the symbol $\sigma$ will indicate the standard deviation of a single measurement, while the value after the symbol \pm will refer to the standard deviation of the mean. 

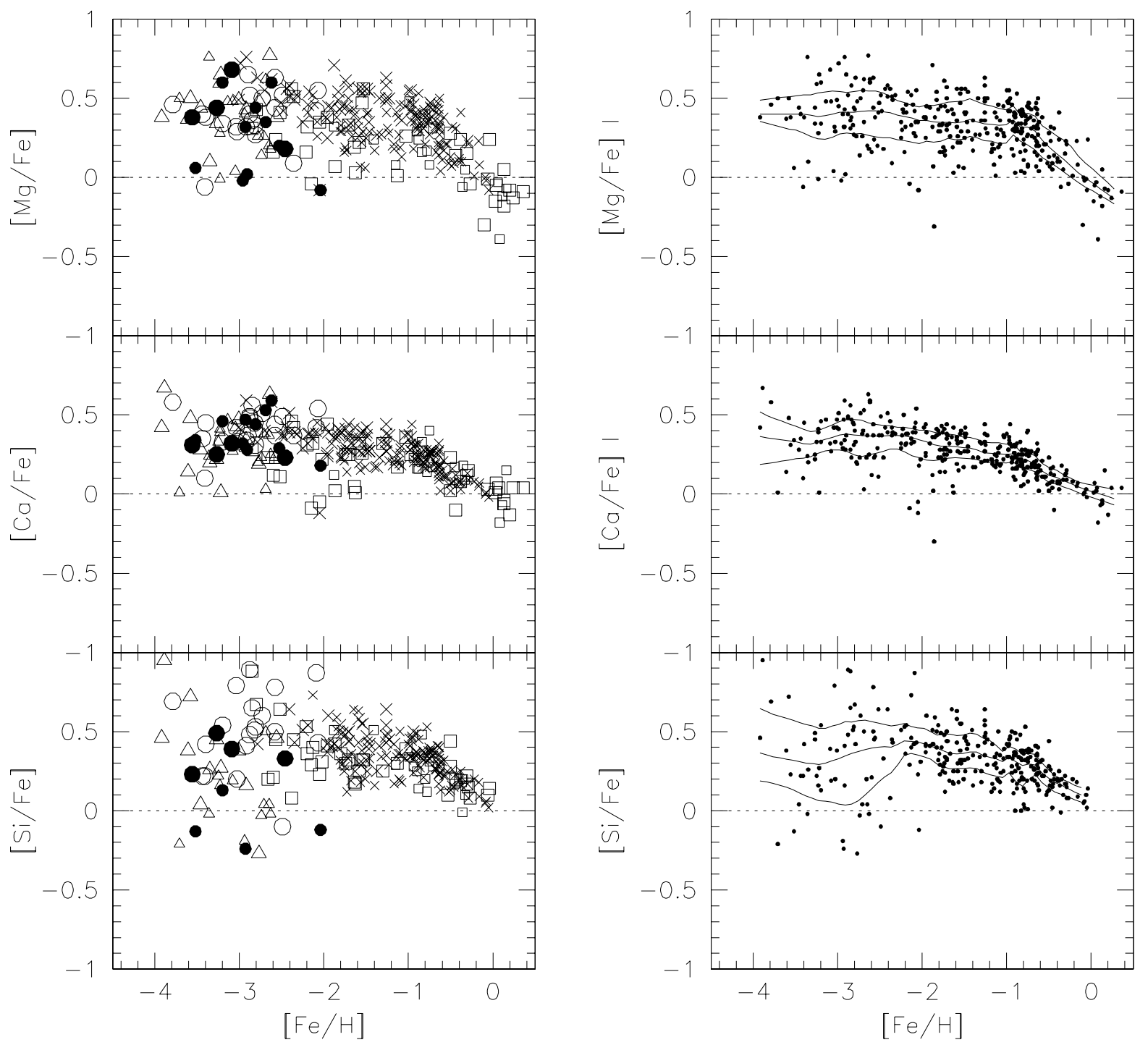

FIG. 6. - Left: Run of the $\alpha$-elements' (Mg, Ca, Si) abundances as a function of metallicity for program stars ( filled circles) as compared with other studies. Open circles, McWilliam et al. (1995) stars; open triangles, Ryan-Norris-Beers (references in Norris et al. 2001); open squares, Gratton samples (see Norris et al. 2001 for references); crosses, Stephens (1999). Larger symbols indicate giant stars, smaller symbols dwarf stars. Right: Data from all the sources listed above and additional data from Gilroy et al. (1988), Carney et al. (1997), Nissen \& Schuster (1997), and Stephens (1999), all displayed as small filled points. The superposed solid lines are the loess lines described in $\S 5.1$

\subsubsection{Aluminum}

The non-LTE aluminum abundances ([Al/Fe $\left.]_{\text {NLTE }}\right)$ for the program stars are shown in Figure 8 (middle) as a function of $[\mathrm{Fe} / \mathrm{H}]$. Aluminum abundances derived without these corrections are shown in the top panel of this figure. In order to make a meaningful comparison, the non-LTE corrections from Baumüller \& Gehren (1997) were applied as well to the abundances in the samples from the literature, also shown in Figure 8, with the same symbols as in previous figures.

Our average $[\mathrm{Al} / \mathrm{Fe}]$ value without correction for nonLTE is $-0.59(\sigma=0.15)$ dex, which is not too far from the mean value as defined at $[\mathrm{Fe} / \mathrm{H}] \sim-3$ dex by the robust trend computed by RNB96 (their Fig. 3c). This means that as far the EWs of these resonance lines are concerned, the agreement between the two different investigations is quite good. Moreover, an offset in the two metallicity scales is not likely to be present, even if we have only one star in com- mon, the well-studied HD 140283. We considered for this star the differences in atmospheric parameters $T_{\text {eff }}, \log g$, and $v_{t}[(5750,3.40,1.40)$, RNB96; and $(5750,3.67,1.10)$, this study]. Reading the changes in $[\mathrm{Fe} / \mathrm{H}]$ associated with these differences from Table 3 of RNB96, the metallicity given by RNB96 $([\mathrm{Fe} / \mathrm{H}]=-2.54 \mathrm{dex})$ would increase by only 0.03 dex. We can say that part of the difference from our value $[\mathrm{Fe} / \mathrm{H}]=-2.44$ dex can be explained by differences in the EWs, and part ( $\sim 0.03$ dex $)$ by a different distribution over excitation potential of the sample of lines used in the analysis.

To compare our results with models of chemical evolution, we adopt, based on theoretical and observational reasons discussed above, the abundances for $\mathrm{Al}$ derived with the non-LTE corrections included. In this case, our average values $[\mathrm{Al} / \mathrm{Fe}]=+0.10(\sigma=0.22)$ and $[\mathrm{Al} / \mathrm{Mg}]=-0.21$ $(\sigma=0.23)$ are not dramatically different from the prediction of models by Timmes, Woosley, \& Weaver (1995). 

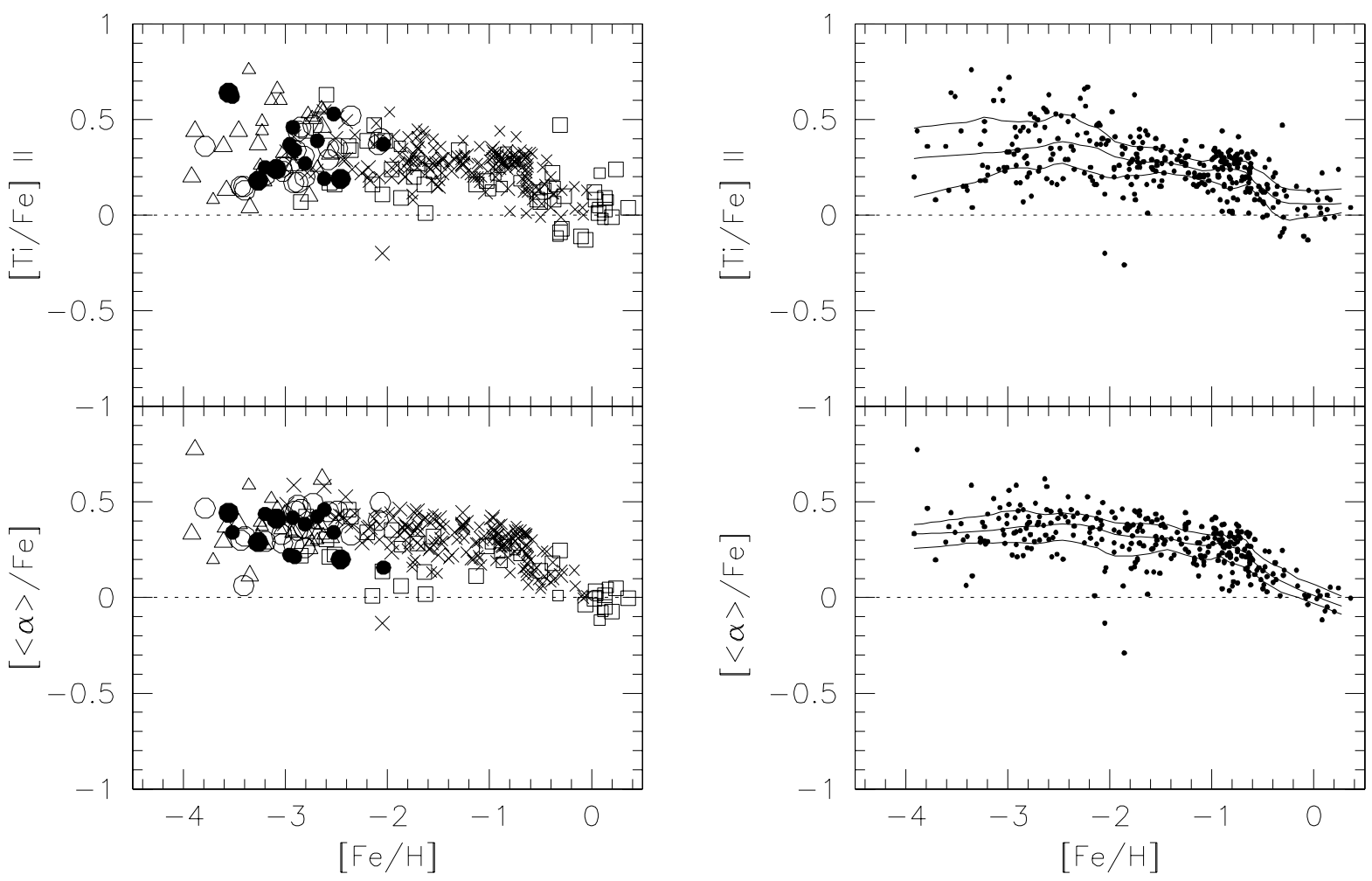

FIG. 7.- Same as Fig. 6, but for Ti II abundances and the average of the $\alpha$-elements, constructed from the abundance ratios of the best-observed ions: Mg I, Ca I, and Ti II.

The case for the sample of McW95 looks quite different. A large scatter in $\mathrm{Al}$ abundances is seen among their stars at each metallicity, and the reason is not obvious. We note that all stars in that sample are giants, and the derivation of atmospheric parameters is somewhat more uncertain for giants than for dwarfs. In conclusion, we have to agree with RNB96 that the higher average $[\mathrm{Al} / \mathrm{Fe}]$ ratio found by McW95 has no obvious explanation.

\subsubsection{The Iron-Peak Elements: $\mathrm{Sc}, \mathrm{Cr}, \mathrm{Mn}$}

Our results for the iron-peak elements are shown in the three left panels of Figure 9. In the region $-2<$ $[\mathrm{Fe} / \mathrm{H}]<-1$, the $\mathrm{Cr}$ and $\mathrm{Mn}$ abundances show a relatively small scatter, as is expected when the contributions from many supernova events average over the initial mass function (IMF) and yields (see Fig. 9). On the other hand, below $[\mathrm{Fe} / \mathrm{H}] \sim-2$ dex the scatter increases, which is characteristic of stochastic models of chemical enrichment. The correlation in the upper panel ( $\mathrm{Cr}$ increasing as $\mathrm{Mn}$ increases) could be explained either if the production of both elements is a function of the mass cut (since they have slightly different atomic number, they are produced in nearby, yet different regions) or if there is a different neutron excess in this metallicity range.

Deeper insight can be obtained looking at Figure 10, where we plot in the top panel the $[\mathrm{Cr} / \mathrm{Fe}]$ ratios versus the abundance ratios $[\mathrm{Mn} / \mathrm{Fe}]$ for stars in the collected "big sample," described in $\S 5.2$, with $[\mathrm{Fe} / \mathrm{H}]<-2$ dex. In this low-metallicity regime, we expect to see the classical signature of Type II SN nucleosynthesis. However, since the neutron excess is known to be a function of metallicity, we can test this hypothesis by removing the trend with metal abun- dance. To this end, we used two linear regressions to fit the $[\mathrm{Cr} / \mathrm{Fe}]$ and $[\mathrm{Mn} / \mathrm{Fe}]$ versus $[\mathrm{Fe} / \mathrm{H}]$ distributions (for stars with $[\mathrm{Fe} / \mathrm{H}]<-2$ dex) and computed the residuals of the abundance ratios with respect to these two fits. The residuals are shown in the bottom panel of Figure 10.

Again, a correlation is evident for both our program stars and for the stars in other samples from literature. Note that the evidence for a correlation holds even if we disregard the extreme case of HE 2344-2800, with its large Mn I abundance. This star has a high-S/N HIRES spectrum, and the high $[\mathrm{Mn} / \mathrm{Fe}]$ ratio is real beyond any doubt: in Figure 11, we compare the spectrum of this star in the region around the Mn lines at 4030-4034 $\AA$ with the spectrum of HE 0024-2523, with very similar atmospheric parameters but no measurable Mn lines.

What do these findings tell us? Following Heger \& Woosley (2002) and Qian \& Wasserburg (2001a, 2001b, 2002), very massive stars (VMSs) that belong to Population III explode as pair-instability $\mathrm{SNe}$ and are expected to produce a $[\mathrm{Cr} / \mathrm{Fe}]$ ratio that is approximately constant, while $[\mathrm{Mn} / \mathrm{Fe}]$ should decrease with metallicity, since nuclei having odd nuclear charge are underproduced by VMSs. Therefore, the signature of VMSs in the iron-peak elements $\mathrm{Cr}$ and $\mathrm{Mn}$ should be a lack of correlation. Since we do observe such a correlation, our results point toward a likely scenario discussed, for example, by Nakamura et al. (1999), who explain the trends of $[\mathrm{Cr} / \mathrm{Fe}]$ and $[\mathrm{Mn} / \mathrm{Fe}]$ ratios decreasing with decreasing $[\mathrm{Fe} / \mathrm{H}]$ as due to a variation of mass cuts in Type II SNe as a function of the progenitor mass. The trends we observe can be reproduced if the mass cut is smaller for the larger mass progenitor, which presumably was the first to evolve and pollute the gas in the early halo. 


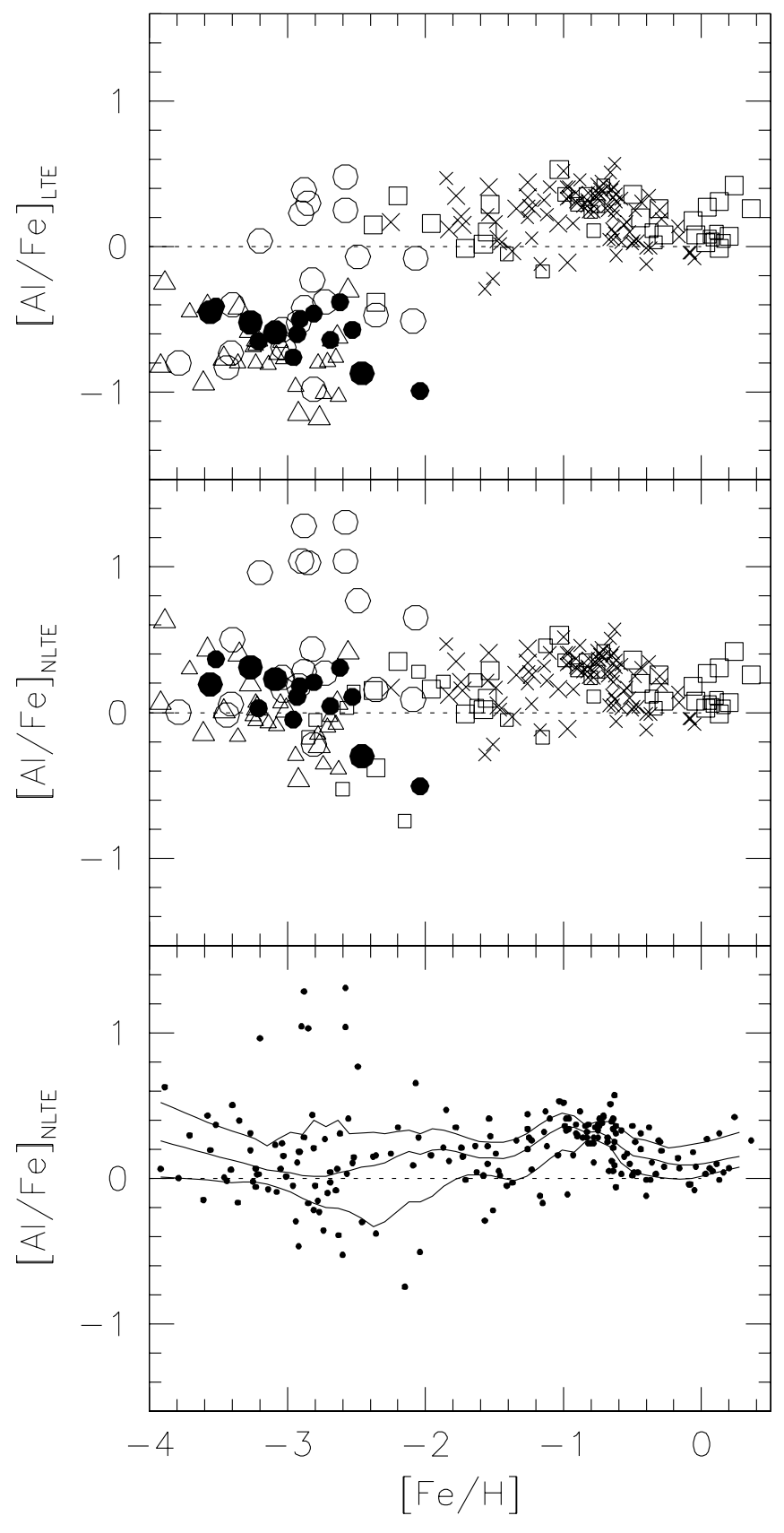

FIG. 8. - Run of $[\mathrm{Al} / \mathrm{Fe}]$ ratios as a function of metallicity. Symbols are as in Fig. 6. Top, $[\mathrm{Al} / \mathrm{Fe}]$ ratios derived in the LTE assumption; middle, $[\mathrm{Al} / \mathrm{Fe}]$ ratios with corrections for departures from LTE, following prescriptions by Baumüller \& Gehren (1997) (see text, § 5.2). Bottom: Abundance ratios $[\mathrm{Al} / \mathrm{Fe}]$ corrected for non-LTE, with the loess summary lines superposed

\subsubsection{Heavy Elements: The Neutron-Capture Elements $\mathrm{Sr}$ and $\mathrm{Ba}$}

Strontium abundances were derived from the $\mathrm{Sr}$ II resonance lines at 4077 and $4215 \AA$, the other accessible lines being vanishingly weak in EMP stars. These strong resonance lines are relatively unaffected by blends in very metalpoor stars and can be easily measured for most of our program stars, excepting those with the lowest $\mathrm{S} / \mathrm{N}$ values. On the other hand, since these are resonance lines, they are saturated in most stars. Hence, the derived $[\mathrm{Sr} / \mathrm{Fe}]$ ratios are sensitive to the details of the stellar parameters and of the adopted model atmosphere (see Table 6). In particular, they are affected by uncertainties in the microturbulent velocity. However, since we derive $v_{t}$ values by using a fair number of Fe $\mathrm{I}$ lines in each star, this is not a serious issue.

Figure 12 presents our results as compared with data from the literature. In this figure, we plot in the top panels the run of $[\mathrm{Sr} / \mathrm{Fe}]$ as a function of the metallicity $[\mathrm{Fe} / \mathrm{H}]$ for our program stars and a compilation of previous studies: McW95 and RNB for very metal-poor dwarfs and giants, and Gratton \& Sneden (1994) for somewhat more metalrich objects. While systematic offsets may be present between different studies (mainly depending on the $T_{\text {eff }}$ scale adopted and on the set of model atmospheres), the overall agreement is fairly good.

Our data confirm once more that at metallicities [Fe/ $\mathrm{H}] \sim-3$ dex (or below -2.4 , the point where most heavyelement patterns show a change in slope; McW95), there is a huge spread in the observed [Sr $/ \mathrm{Fe}]$ values, reaching almost 3 dex. This scatter is not linked to a particular evolutionary stage, being present among both dwarf and giant stars. The increase in scatter for $[\mathrm{Sr} / \mathrm{Fe}]$ values with decreasing metallicity is commonly explained by the classical enrichment of $r$-process elements from explosions of massive Type II SNe (above 12-15 $M_{\odot}$ ) in the framework of a stochastic enrichment mechanism (McW95). In this scenario, if only few SNe contribute to the production of $\mathrm{Sr}$, we expect a strongly asymmetric distribution in the logarithmic plane $[\mathrm{Sr} / \mathrm{Fe}]$ versus $[\mathrm{Fe} / \mathrm{H}]$, with more stars with low $[\mathrm{Sr} / \mathrm{Fe}]$ than stars with high $\mathrm{Sr}$.

The $r$-enhanced region $([\mathrm{Sr} / \mathrm{Fe}]>0$ in the top panels of Fig. 12) is attributed to the same classical site of $r$-process production, but restricted in this case to very few SNe less massive than 12-15 $M_{\odot}$. As a consequence of the limited number of objects contributing to the enrichment, the whole process has a strongly stochastic behavior, revealed in a few stars with abnormally high $r$-process abundances.

Barium abundances were derived from the $\mathrm{Ba}$ II resonance line at $4554.0 \AA$. This line has appreciable HFS, taken into account when computing the abundances of Table 5 by using data from Steffen (1985). Figure 12 (bottom) summarizes and compares our results with previous studies. Apart from the few objects above $[\mathrm{Ba} / \mathrm{Fe}] \sim 1$ (whose origin is somewhat different; see below), Ba shows a decrease for $[\mathrm{Fe} / \mathrm{H}]<-2$ dex and less scatter than the lighter $n$-capture element $\mathrm{Sr}$. The main features of this figure are commonly explained by the two classical sources of Ba production: the main $s$-process in intermediate-mass stars (4-7 $\left.M_{\odot}\right)$ evolving through the asymptotic giant branch (AGB) phase, plus a contribution by the $r$-process in $\mathrm{SNe}$. As a consequence of the evolutionary stellar timescales involved, what is seen below $[\mathrm{Fe} / \mathrm{H}]=-2$ dex is essentially the enrichment of $r$ elements from massive Type II SNe, whose entire evolution from birth to death is much quicker than the timescale required for lower mass stars to reach the AGB and for complete mixing of the winds from these stars.

Finally, Figure 13 shows the relation between the abundances of $\mathrm{Sr}$ and $\mathrm{Ba}$ - these elements are often chosen to represent the behavior of light and heavy $n$-capture elements, respectively. This plot is quite instructive, since we can see that for the majority of the stars, the $[\mathrm{Sr} / \mathrm{Fe}]$ and $[\mathrm{Ba} / \mathrm{Fe}]$ ratios lie almost exactly on a line that is simply that of a scaled solar composition. The low-Sr, low-Ba region reflects the classical $r$-enrichment by massive SNe. However, in this figure we can also see a sort of branch or plume of stars having a high $\mathrm{Sr}$ content, and proportionally less $\mathrm{Ba}$. 

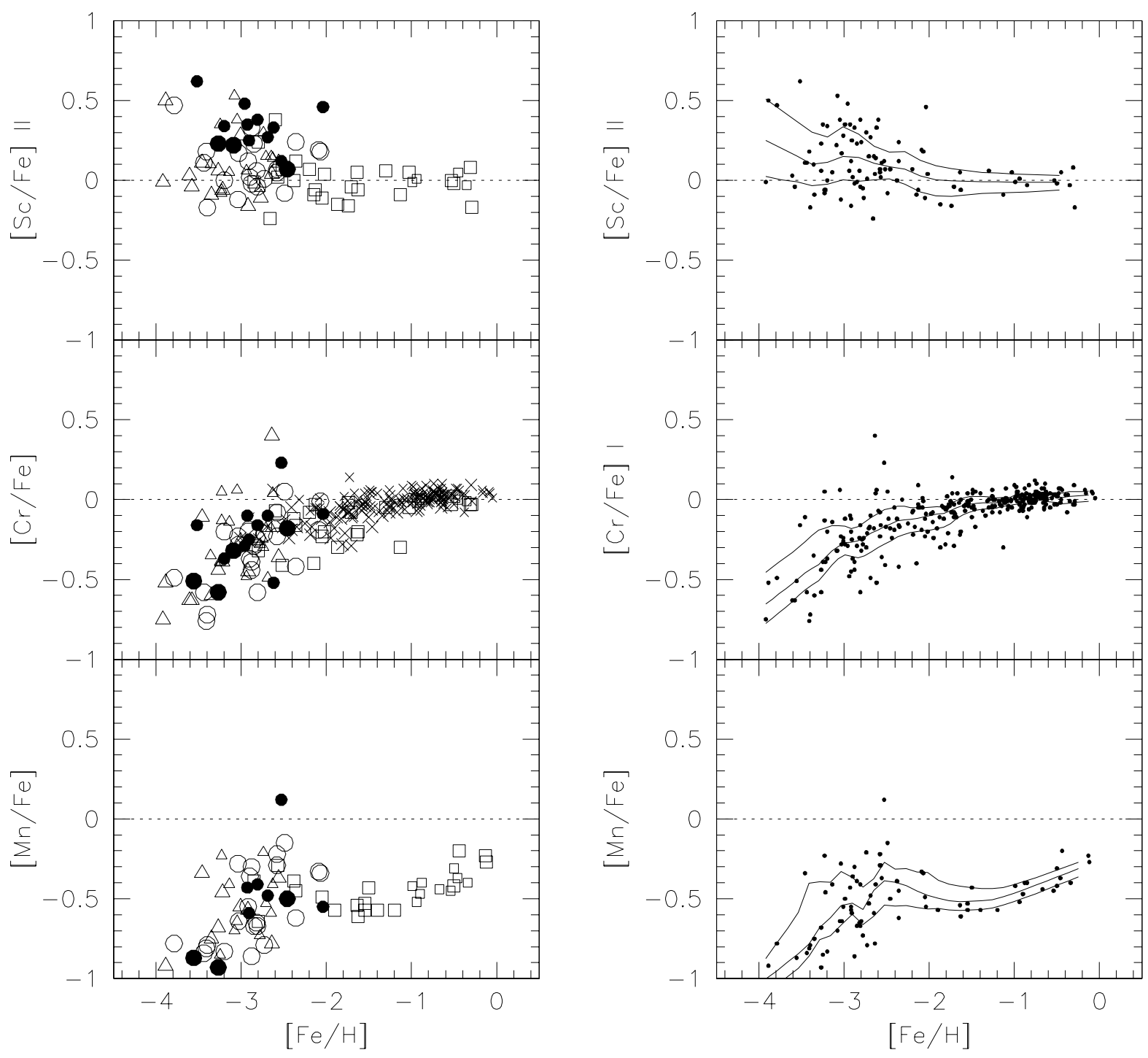

FIG. 9.-Abundances of iron-peak elements in program stars as a function of [Fe/H], as compared with data from previous studies, as in Fig. 6. The left panels allow one to differentiate among data sets, while the right panels, with summary lines superposed, plot all data with a single symbol irrespective of source.

This group of stars seems to lie outside the general trend. If confirmed, this could imply that a unique site of $r$-process production might not be not sufficient, and we would require an additional nucleosynthetic mechanism able to provide almost exclusively light $n$-capture elements (such as $\mathrm{Sr}$ ), with only a small amount of heavier $n$-capture elements (such as Ba). This idea is not new (Wasserburg \& Qian 2000), but it will require a much larger sample of stars, analyzed in a consistent and homogeneous manner, in order to be tested.

\subsection{A" Big" Sample}

The right panels in Figures 6, 7, 9, and 12, as well as the bottom panel in Figure 8, show all the data displayed in the left panels as small circles, regardless of their source. Also, additional data were added from other studies that were not included in the left panels to maintain the clarity and avoid overcrowding of the latter. Following NRB01, we add into these right panels data from Gilroy et al. (1988), Nissen \&
Schuster (1997), Carney et al. (1997), and Stephens (1999). ${ }^{12}$ Superposed in these panels are lines indicating abundance trends determined with robust statistical tools. The summary lines in these figures are described in detail in NRB01; we present a brief description below.

The summary lines are robust locally weighted regression lines (abbreviated as loess lines), described by Cleveland $(1979,1994)^{13}$ and determined as follows: First, average values of each abundance ratio were obtained. Next we obtained three summary lines - the central loess line (CLL),

\footnotetext{
${ }^{12}$ NRB01 made an effort in their paper to bring onto a homogeneous system the previous data from the literature that they used to build up their comparison sample. However, unless the measured EWs are reanalyzed in the same fashion, using the same model atmospheres and the same procedure to derive atmospheric parameters, we cannot exclude the possibility that residual systematic (small) offsets are still present among different samples.

${ }^{13}$ The source code for loess regression can be obtained from http:// www.astro.psu/edu/statcodes/sc_correlregr.html. It is also available as a regression option in many commercially available statistical packages.
} 

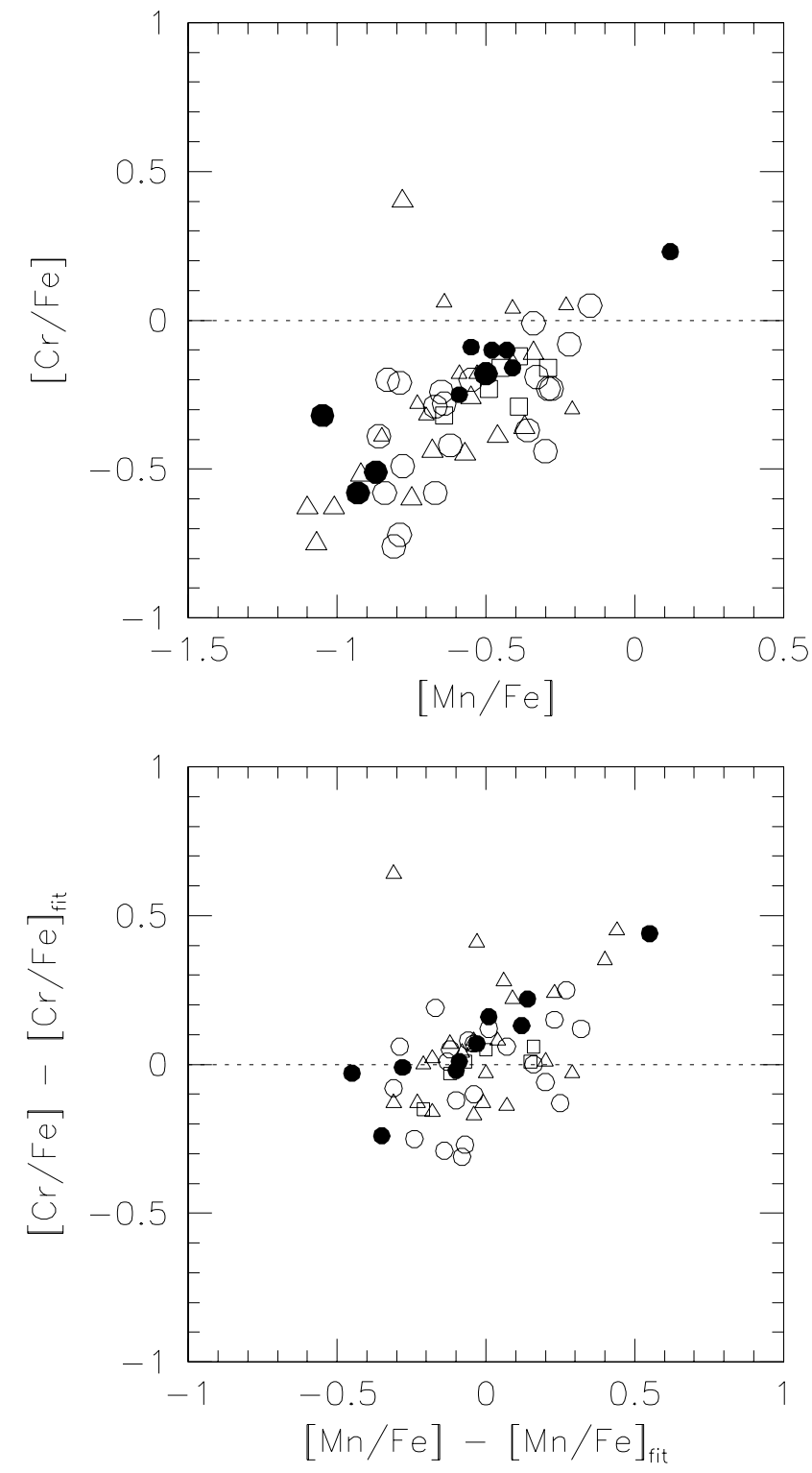

FIG. 10.-Top: Run of the $[\mathrm{Cr} / \mathrm{Fe}]$ abundance ratio as a function of $[\mathrm{Mn} / \mathrm{Fe}]$ for program stars and for data from the literature. Symbols are as in Fig. 6. Only stars with $[\mathrm{Fe} / \mathrm{H}]<-2$ dex are plotted. Bottom: Residuals of $[\mathrm{Cr} / \mathrm{Fe}]$ and $[\mathrm{Mn} / \mathrm{Fe}]$ ratios with respect to linear regressions obtained from $[\mathrm{Cr} / \mathrm{Fe}]$ vs. $[\mathrm{Fe} / \mathrm{H}]$ and $[\mathrm{Mn} / \mathrm{Fe}]$ vs. $[\mathrm{Fe} / \mathrm{H}]$ diagrams for stars with $[\mathrm{Fe} / \mathrm{H}]<-2$ dex.

the lower loess line (LLL), and the upper loess line (ULL) as a function of the $[\mathrm{Fe} / \mathrm{H}]$ values. The CLL is defined as the loess line when all the data are considered and provides our best estimate of the general trend of the elemental ratios at a given $[\mathrm{Fe} / \mathrm{H}]$. Next, residuals about the CLL were obtained and separated into those above (positive residuals) and below (negative residuals) this line. The LLL is defined as the loess line for the negative residuals as a function of $[\mathrm{Fe} / \mathrm{H}]$. The ULL is defined as the loess line for the positive residuals as a function of $[\mathrm{Fe} / \mathrm{H}]$. If the data are scattered about the CLL according to a normal distribution, then the LLL and ULL are estimates of the true quartiles. The loess lines remain sensitive to local variations without being unduly influenced by outliers. Furthermore, they are able to better handle the endpoints of the data sets than the more

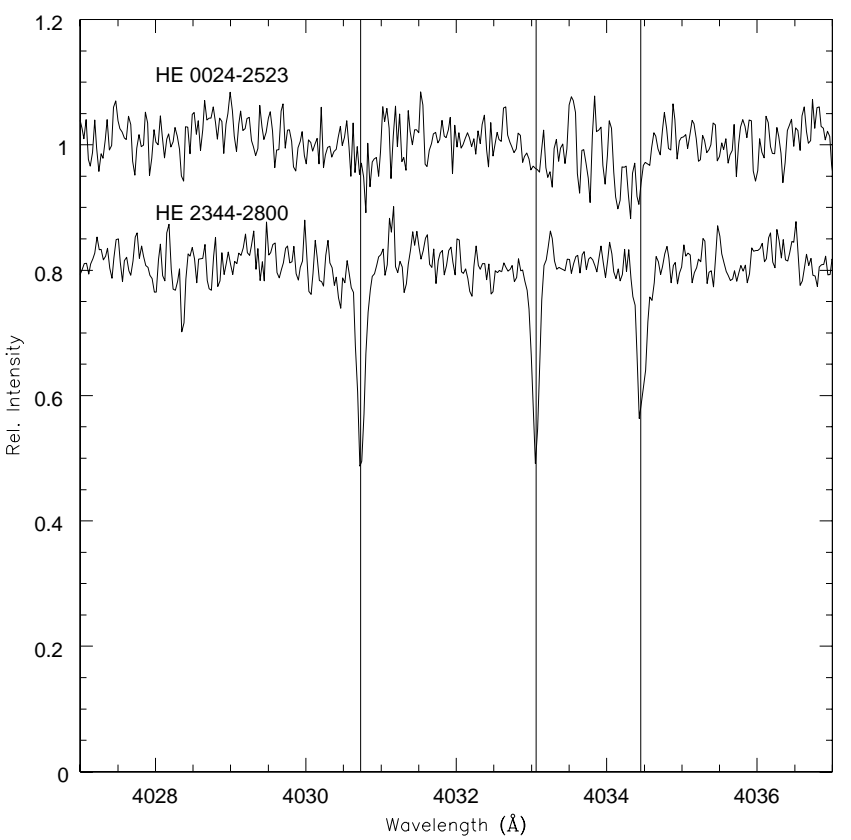

FIG. 11.-Observed spectra of the program stars HE 0024-2523 and HE 2344-2800 in the region around the Mn I lines at 4030.75, 4033.06, and $4034.48 \AA$ (vertical lines). The spectrum of HE 2344-2800 has been arbitrarily shifted in intensity for purposes of clarity. Note that HE 2344-2800 is the object with the anomalously large Mn abundance in Fig. 10.

commonly used median lines. In each panel, the CLL is flanked by the ULL and CLL.

In order to quantify the abundance scatter in these diagrams, we compute the scale ${ }^{14}$ of the data for each elemental ratio, making use of the CLL obtained above, and consider the complete set of residuals in the ordinate of each data point about the trend. In Table 7, we summarize robust estimates of the scale of these residuals over several ranges in $[\mathrm{Fe} / \mathrm{H}]$, using the biweight estimator of scale, $S_{\mathrm{BI}}$, described by Beers, Flynn, \& Gebhardt (1990). The first column of the table lists the abundance ranges considered. In setting these ranges, we sought to maintain a minimum bin population of $N=15-20$. The second column lists the mean $[\mathrm{Fe} / \mathrm{H}]$ of the stars in the listed abundance interval, and the third lists the numbers of stars contained in that interval. The fourth column lists $S_{\mathrm{BI}}$, along with error bars obtained by analysis of 1000 bootstrap resamplings of the data in the bin. These errors are useful for assessing the significance of the difference between the scales of the data from bin to bin. Note that with the inclusion of the newly measured data from our present paper, as well as from other recent sources, we are able to provide scatter estimates for a somewhat finer grid, extending to lower metallicities, than was presented by NRB01.

\subsection{The Scatter in the $[\mathrm{Mg} / \mathrm{Fe}]$ Ratio and Its Interpretation}

The intrinsic scatter in the element-to-element ratios at various metallicities contains valuable information about the typical size of the clouds undergoing independent chemical evolution in the early epochs of halo formation, as well as on the typical number of SNe that polluted such clouds. For the purpose of this discussion, we assume that only

\footnotetext{
14 The scale matches the dispersion for a normal distribution.
} 

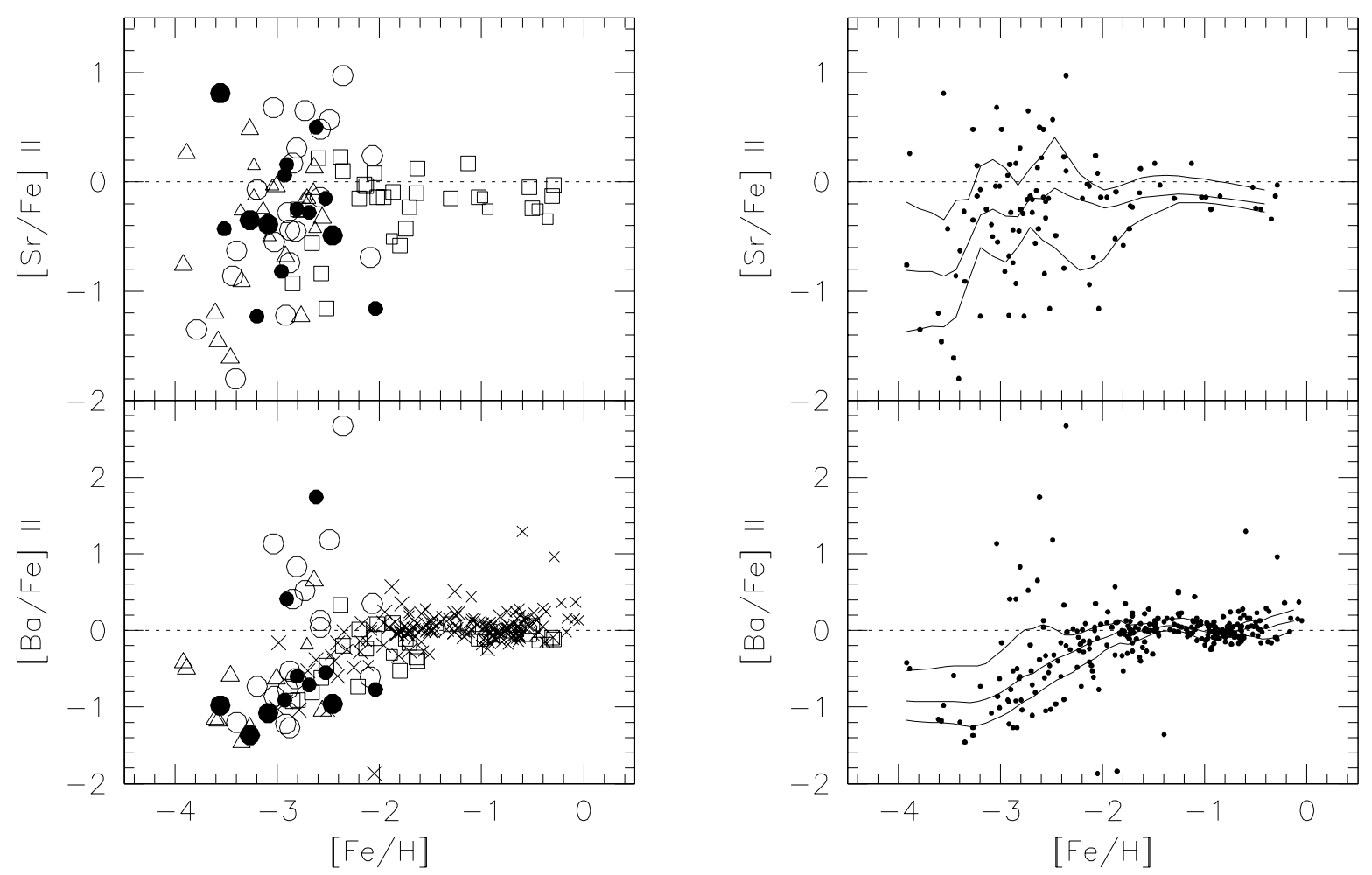

FIG. 12. - Comparison of the run of elemental ratios of the $n$-capture elements $\mathrm{Sr}(t o p)$ and $\mathrm{Ba}($ bottom $)$ as a function of [Fe/H] in the present work and other previous studies; the symbols have the same meaning as in previous figures.

core-collapse $\mathrm{SNe}$ are important contributors to element production in this early phase of the Galaxy. We will return later to this point, to briefly comment on the possible impact of nucleosynthesis from VMSs.

The most interesting elements in the present context are $\mathrm{Fe}$ (assumed to be representative of the abundance of Fe-

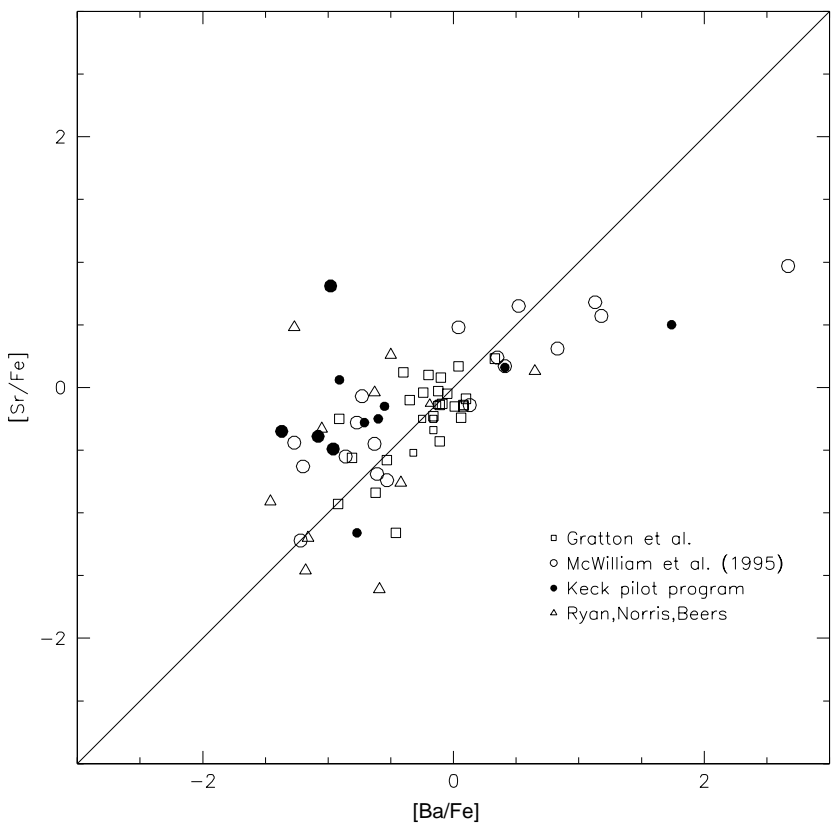

FIG. 13. - The light $n$-capture element $[\mathrm{Sr} / \mathrm{Fe}]$ as a function of the heavier $n$-capture element $[\mathrm{Ba} / \mathrm{Fe}]$. peak elements) and the $\alpha$-elements, because the ratio in an EMP star of the abundance of Fe to that of the $\alpha$-elements is expected to be quite sensitive to the original mass of the $\mathrm{SN}$. Important information is also provided by other elements (such as those produced by rapid $n$-capture); these have been considered by other authors (see, e.g., McWilliam 1997; Qian \& Wasserburg 2001a, 2001b, 2002). However, it is possible that only $\mathrm{SNe}$ with progenitors in a restricted mass range have contributed significantly to the production of many of these other elements, and the exact mass ranges are not known at present.

We concentrate here on the $\mathrm{Mg} / \mathrm{Fe}$ ratio, since this is available for a large number of stars with $[\mathrm{Fe} / \mathrm{H}]<-2$ dex, is not overly sensitive to the details of the abundance analysis (a major concern for oxygen; see, e.g., Asplund \& García Pérez 2001), and is less sensitive to details of nucleosynthesis than ratios involving other elements, for example, $\mathrm{Ca}$ and Ti. From the numbers given in the previous subsection, we note that the rms scatter for the ratio $[\mathrm{Mg} / \mathrm{Fe}]$ over the available sample of EMP stars is $0.19 \pm 0.03$ near $[\mathrm{Fe} / \mathrm{H}]=-3$ dex, and $0.16 \pm 0.03$ near $[\mathrm{Fe} / \mathrm{H}]=-2$ dex. Part of this scatter must arise from problems in the observations and analysis, rather than being intrinsic. This is certainly the case for another element of interest, $\mathrm{Si}$, for which the analogous values are $0.37 \pm 0.05$ and $0.13 \pm 0.02$. The large scatter at very low metallicities of the $[\mathrm{Si} / \mathrm{Fe}]$ ratios can be attributed to the fact that $\mathrm{Si}$ abundances in EMP stars are usually derived from a single line (at $3905 \AA$ ) and are thus very uncertain. However, in the case of $\mathrm{Mg}$ the observational constraints are less severe, as there are several clean lines of $\mathrm{Mg}$ I that are strong enough to be detectable in EMP stars. Since we are only interested here in order-ofmagnitude estimates, we will ignore any noncosmic scatter 
TABLE 7

Robust Scatter Estimates for Elemental Ratios in Metal-poor Stars

\begin{tabular}{|c|c|c|c|c|c|c|c|}
\hline $\begin{array}{l}{[\mathrm{Fe} / \mathrm{H}] \text { Range }} \\
(\mathrm{dex})\end{array}$ & $\langle\mathrm{Fe} / \mathrm{H}\rangle$ & $N$ & Mean and $\sigma^{\mathrm{a}}$ & $\begin{array}{c}{[\mathrm{Fe} / \mathrm{H}] \text { Range }} \\
(\mathrm{dex})\end{array}$ & $\langle\mathrm{Fe} / \mathrm{H}\rangle$ & $N$ & Mean and $\sigma^{\mathrm{a}}$ \\
\hline$[\mathrm{Mg} / \mathrm{Fe}]:$ & & & & {$[\mathrm{Al} / \mathrm{Fe}]($ non-LTE): } & & & \\
\hline$>-0.5 \ldots \ldots \ldots \ldots$ & -0.177 & 39 & $0.125_{-0.013}^{+0.026}$ & $>-0.5 \ldots \ldots \ldots \ldots$ & -0.156 & 34 & $0.131_{-0.012}^{+0.016}$ \\
\hline-1.0 to $-0.5 \ldots \ldots$ & -0.769 & 91 & $0.124_{-0.008}^{+0.012}$ & -1.0 to $-0.5 \ldots \ldots$ & -0.750 & 57 & $0.123_{-0.015}^{+0.017}$ \\
\hline-1.5 to $-1.0 \ldots \ldots$ & -1.231 & 42 & $0.147_{-0.011}^{+0.017}$ & -1.5 to $-1.0 \ldots \ldots$. & -1.234 & 21 & $0.186_{-0.027}^{+0.046}$ \\
\hline-2.0 to $-1.5 \ldots \ldots$ & -1.720 & 54 & $0.161_{-0.018}^{+0.021}$ & -2.5 to $-1.5 \ldots \ldots$ & -1.913 & 29 & $0.286_{-0.059}^{+0.098}$ \\
\hline-2.5 to $-2.0 \ldots \ldots$ & -2.233 & 33 & $0.170_{-0.030}^{+0.041}$ & -3.0 to $-2.5 \ldots \ldots$ & -2.758 & 34 & $0.410_{-0.085}^{+0.156}$ \\
\hline-3.0 to $-2.5 \ldots \ldots$ & -2.761 & 42 & $0.193_{-0.017}^{+0.024}$ & $\leq-3.0 \ldots \ldots \ldots \ldots$ & -3.348 & 29 & $0.209_{-0.030}^{+0.047}$ \\
\hline$\leq-3.0 \ldots \ldots \ldots \ldots$ & -3.337 & 30 & $0.193_{-0.040}^{+0.055}$ & {$[\mathrm{Al} / \mathrm{Mg}]($ non-LTE): } & & & \\
\hline$[\mathrm{Ca} / \mathrm{Fe}]:$ & & & & $>-0.5 \ldots \ldots \ldots \ldots \ldots$ & -0.156 & 34 & $0.121_{-0.017}^{+0.020}$ \\
\hline$>-0.5 \ldots \ldots \ldots \ldots$ & -0.183 & 39 & $0.062_{-0.008}^{+0.015}$ & -1.0 to $-0.5 \ldots \ldots$ & -0.750 & 57 & $0.076_{-0.011}^{+0.014}$ \\
\hline-1.0 to $-0.5 \ldots \ldots$ & -0.768 & 92 & $0.075_{-0.006}^{+0.007}$ & -1.5 to $-1.0 \ldots \ldots$ & -1.234 & 21 & $0.110_{-0.021}^{+0.069}$ \\
\hline-1.5 to $-1.0 \ldots \ldots$ & -1.231 & 41 & $0.081_{-0.006}^{+0.009}$ & -2.5 to $-1.5 \ldots \ldots$ & -1.913 & 29 & $0.253_{-0.030}^{+0.053}$ \\
\hline-2.0 to $-1.5 \ldots \ldots$ & -1.724 & 54 & $0.108_{-0.011}^{+0.019}$ & -3.0 to $-2.5 \ldots \ldots$ & -2.758 & 34 & $0.388_{-0.072}^{+0.106}$ \\
\hline-2.5 to $-2.0 \ldots \ldots$ & -2.221 & 34 & $0.086_{-0.017}^{+0.027}$ & $\leq-3.0 \ldots \ldots \ldots \ldots$ & -3.348 & 29 & $0.337_{-0.038}^{+0.068}$ \\
\hline-3.0 to $-2.5 \ldots \ldots$ & -2.759 & 43 & $0.144_{-0.012}^{+0.016}$ & {$[\mathrm{Sc} / \mathrm{Fe}]:$} & & & \\
\hline$\leq-3.0 \ldots \ldots \ldots \ldots$ & -3.337 & 30 & $0.133_{-0.023}^{+0.035}$ & $>-1.5 \ldots \ldots \ldots \ldots$ & -0.723 & 12 & $0.066_{-0.012}^{+0.030}$ \\
\hline$[\mathrm{Si} / \mathrm{Fe}]:$ & & & & -2.5 to $-1.5 \ldots \ldots$ & -2.077 & 18 & $0.138_{-0.022}^{+0.057}$ \\
\hline$>-0.5 \ldots \ldots \ldots \ldots \ldots$ & -0.314 & 31 & $0.062_{-0.007}^{+0.016}$ & -3.0 to $-2.5 \ldots \ldots$ & -2.757 & 34 & $0.176_{-0.014}^{+0.024}$ \\
\hline-1.0 to $-0.5 \ldots \ldots$ & -0.766 & 94 & $0.111_{-0.009}^{+0.012}$ & $\leq-3.0 \ldots \ldots \ldots \ldots$ & -3.335 & 28 & $0.233_{-0.028}^{+0.076}$ \\
\hline-1.5 to $-1.0 \ldots \ldots$ & -1.233 & 43 & $0.128_{-0.012}^{+0.014}$ & {$[\mathrm{Cr} / \mathrm{Fe}]:$} & & & \\
\hline-2.0 to $-1.5 \ldots \ldots$ & -1.712 & 52 & $0.131_{-0.010}^{+0.013}$ & $>-0.5 \ldots \ldots \ldots \ldots$ & -0.330 & 21 & $0.043_{-0.005}^{+0.010}$ \\
\hline-2.5 to $-2.0 \ldots \ldots$ & -2.211 & 20 & $0.226_{-0.054}^{+0.067}$ & -1.0 to $-0.5 \ldots \ldots$ & -0.771 & 87 & $0.040_{-0.003}^{+0.004}$ \\
\hline-3.0 to $-2.5 \ldots \ldots$ & -2.751 & 27 & $0.368_{-0.038}^{+0.048}$ & -1.5 to $-1.0 \ldots \ldots$ & -1.227 & 40 & $0.050_{-0.005}^{+0.010}$ \\
\hline$\leq-3.0 \ldots \ldots \ldots \ldots$ & -3.372 & 26 & $0.267_{-0.040}^{+0.060}$ & -2.0 to $-1.5 \ldots \ldots$. & -1.722 & 46 & $0.076_{-0.016}^{+0.019}$ \\
\hline$[\mathrm{Ti} / \mathrm{Fe}]:$ & & & & -2.5 to $-2.0 \ldots \ldots$ & -2.207 & 29 & $0.111_{-0.015}^{+0.029}$ \\
\hline$>-0.5 \ldots \ldots \ldots \ldots$ & -0.183 & 41 & $0.105_{-0.012}^{+0.018}$ & -3.0 to $-2.5 \ldots \ldots$. & -2.759 & 41 & $0.167_{-0.025}^{+0.034}$ \\
\hline-1.0 to $-0.5 \ldots \ldots$ & -0.766 & 91 & $0.092_{-0.007}^{+0.010}$ & $\leq-3.0$ & -3.324 & 29 & $0.186_{-0.028}^{+0.031}$ \\
\hline-1.5 to $-1.0 \ldots \ldots$ & -1.231 & 41 & $0.061_{-0.005}^{+0.008}$ & {$[\mathrm{Mn} / \mathrm{Fe}]:$} & & & \\
\hline-2.0 to $-1.5 \ldots \ldots$ & -1.729 & 52 & $0.117_{-0.010}^{+0.022}$ & $>-1.5 \ldots \ldots \ldots \ldots \ldots$ & -0.679 & 16 & $0.065_{-0.008}^{+0.021}$ \\
\hline-2.5 to $-2.0 \ldots \ldots$ & -2.226 & 35 & $0.166_{-0.015}^{+0.030}$ & -2.5 to $-1.5 \ldots \ldots$ & -2.005 & 15 & $0.115_{-0.023}^{+0.038}$ \\
\hline-3.0 to $-2.5 \ldots \ldots$ & -2.759 & 41 & $0.154_{-0.012}^{+0.019}$ & -3.0 to $-2.5 \ldots \ldots$ & -2.765 & 27 & $0.184_{-0.025}^{+0.045}$ \\
\hline$\leq-3.0 \ldots \ldots \ldots \ldots$ & -3.337 & 30 & $0.212_{-0.023}^{+0.027}$ & $\leq-3.0$ & -3.331 & 25 & $0.205_{-0.038}^{+0.048}$ \\
\hline$[\langle\alpha\rangle / \mathrm{Fe}]:$ & & & & {$[\mathrm{Sr} / \mathrm{Fe}]:$} & & & \\
\hline$>-0.5 \ldots \ldots \ldots \ldots \ldots$ & -0.187 & 44 & $0.079_{-0.009}^{+0.015}$ & $>-1.5 \ldots \ldots \ldots \ldots \ldots$ & -0.829 & 14 & $0.143_{-0.021}^{+0.031}$ \\
\hline-1.0 to $-0.5 \ldots \ldots$. & -0.766 & 94 & $0.087_{-0.006}^{+0.008}$ & -2.5 to $-1.5 \ldots \ldots$ & -2.053 & 24 & $0.458_{-0.068}^{+0.112}$ \\
\hline-1.5 to $-1.0 \ldots \ldots$. & -1.233 & 43 & $0.083_{-0.007}^{+0.010}$ & -3.0 to $-2.5 \ldots \ldots$. & -2.744 & 35 & $0.492_{-0.058}^{+0.071}$ \\
\hline-2.0 to $-1.5 \ldots \ldots$ & -1.721 & 55 & $0.114_{-0.011}^{+0.017}$ & $\leq-3.0 \ldots \ldots \ldots$ & -3.343 & 26 & $0.664_{-0.088}^{+0.145}$ \\
\hline-2.5 to $-2.0 \ldots \ldots$ & -2.226 & 36 & $0.106_{-0.022}^{+0.034}$ & {$[\mathrm{Ba} / \mathrm{Fe}]:$} & & & \\
\hline-3.0 to $-2.5 \ldots \ldots$ & -2.759 & 43 & $0.112_{-0.011}^{+0.013}$ & $>-0.5 \ldots \ldots \ldots$ & -0.328 & 23 & $0.184_{-0.022}^{+0.080}$ \\
\hline$\leq-3.0 \ldots \ldots \ldots \ldots$ & -3.337 & 30 & $0.101_{-0.022}^{+0.029}$ & -1.0 to $-0.5 \ldots \ldots$ & -0.767 & 89 & $0.097_{-0.007}^{+0.012}$ \\
\hline$[\mathrm{Al} / \mathrm{Fe}](\mathrm{LTE}):$ & & & & -1.5 to $-1.0 \ldots \ldots$. & -1.242 & 43 & $0.127_{-0.017}^{+0.030}$ \\
\hline$>-0.5 \ldots \ldots \ldots \ldots$ & -0.156 & 34 & $0.131_{-0.012}^{+0.016}$ & -2.0 to $-1.5 \ldots \ldots$ & -1.720 & 52 & $0.201_{-0.024}^{+0.034}$ \\
\hline-1.0 to $-0.5 \ldots \ldots$ & -0.750 & 57 & $0.124_{-0.014}^{+0.018}$ & -2.5 to $-2.0 \ldots \ldots$ & -2.229 & 37 & $0.338_{-0.059}^{+0.075}$ \\
\hline-1.5 to $-1.0 \ldots \ldots$ & -1.234 & 21 & $0.204_{-0.032}^{+0.037}$ & -3.0 to $-2.5 \ldots \ldots$ & -2.741 & 34 & $0.611_{-0.119}^{+0.156}$ \\
\hline-2.5 to $-1.5 \ldots \ldots$ & -1.913 & 29 & $0.426_{-0.043}^{+0.085}$ & $\leq-3.0 \ldots \ldots \ldots \ldots$ & -3.353 & 16 & $0.366_{-0.054}^{+0.158}$ \\
\hline-3.0 to $-2.5 \ldots \ldots$ & -2.758 & 34 & $0.428_{-0.076}^{+0.094}$ & & & & \\
\hline$\leq-3.0 \ldots \ldots \ldots \ldots$ & -3.348 & 29 & $0.202_{-0.021}^{+0.057}$ & & & & \\
\hline
\end{tabular}

NoтE.- This table was constructed from the results of the high-dispersion analyses in the papers quoted in $\S \S 5.1 .2$ and 5.2. In the case of multiple analyses for the same star, we have averaged the results.

a Mean and dispersion calculated using the biweight estimators of Beers et al. 1990, as they are very robust to the presence of outliers. See text for additional information. 
(e.g., due to use of subsamples from other studies) in the $\mathrm{Fe}$ and $\mathrm{Mg}$ abundances, as we anticipate that measured values for the $\mathrm{Mg} / \mathrm{Fe}$ scatter are already quite small with respect to expectations, so that any further reduction would strengthen our conclusion.

Comprehensive treatments of the scatter in element-toelement abundances among EMP stars have been recently produced by various authors (see, e.g., Argast et al. 2000; Karlsson \& Gustafsson 2001; references therein). Models that take into account the stochastic effect of pollution from individual $\mathrm{SNe}$, as well as the lifetimes of their progenitors, have been developed. Such models allow a detailed description of the interplay between stellar evolutionary times and the time required for a complete mixing of a suitable fragment of the original halo. However, such modeling requires various input quantities (e.g., yields) that are not well known at present, so conclusions can only be reached at order-of-magnitude levels. Furthermore, it is not entirely clear that the models adequately reproduce the mechanisms of star formation and mixing within the interstellar medium (ISM) (e.g., stars are considered to form individually, rather than in clusters). In the following, we offer a much simpler approach that allows easy insight into some important issues while still maintaining order-of-magnitude accuracy. We invite the reader to consider all the following results as very preliminary and model dependent. Once a better understanding of the basic physics becomes available, complex models such as those considered by Argast et al. (2000) or Karlsson \& Gustafsson (2001) must be considered, with possibly an even more elaborate formulation for the star formation process and for mixing within the ISM.

The essential ideas of our approach are to consider the early Galaxy as made of several independent clouds and to treat each cloud as a closed box ${ }^{15}$ undergoing its own chemical evolution. We further assume that the ISM of each protogalactic cloud, from which the EMP stars we currently observe formed, was metal-enriched by the ejecta of $\mathrm{SNe}$ produced by a single generation of progenitors. Of course, this is a very schematic approach but, we think, still useful; it might correspond to a picture in which a small star cluster or association begins forming within a primordial cloud (still with zero metals). The winds and SN ejecta from its most massive stars pollute the remaining part of the cloud, from which a second generation of stars (those we currently observe) form. Effective mixing due to turbulence is assumed to maintain the chemical homogeneity of the cloud (note that relaxing this condition would increase the expected scatter in the element-to-element ratios). In a closed-box model, the metal abundance of the ISM is set by the yields and by the fraction of gas still remaining. Hence, once the yields are known (from SN models and from assumption of an IMF) the fraction of gas remaining is unequivocally determined. The next step is to insert discreteness, that is, a finite number of SNe. If $\mathrm{SN}$ yields for individual elements are not constant and depend, for exam-

\footnotetext{
${ }^{15}$ In the terminology of chemical evolution models, a "closed-box model" is a model in which there is no exchange of matter with external components (that is, neither infall nor outflow of matter). Closed-box models are very simple (see, e.g., Pagel 1997). An important property of the closed-box models is the simple relation existing between the concentration of metal $i$ in the gas $z_{i}$ and the fraction of matter still in gas form $g$ : $z_{i}=y_{i} \ln g$, where $y_{i}$ is the yield of the metal $i$ through production in stellar interiors.
}

ple, on the initial stellar mass, we should expect a scatter in element-to-element abundances obtained from different clouds, depending on the particular set of $\mathrm{SNe}$ that exploded in a given cloud. As a consequence of Poisson statistics, we expect that the scatter will be a function of the actual number of SNe contributing to typical clouds. Since the number of $\mathrm{SNe}$ for a given total mass is fixed by the IMF, it is possible to normalize the total mass in stars and, from the fraction of gas (given by the overall metallicity), to derive the total mass of the cloud.

The above model can easily be simulated by using an appropriate Monte Carlo code. Essentially, we need to assume an IMF (here we used the Miller \& Scalo 1979 IMF; note that the low-mass cutoff of the IMF is not critical here, only affecting the number of low-mass stars of the very first generation expected to still exist on the lower main sequence at present) and yields for different elements as a function of mass. These are by far the most uncertain quantities at present; the yield of $\mathrm{Fe}$ is particularly uncertain, as it strongly depends on the assumed mass cut in the SN model, a poorly known quantity. Current SN models are unable to provide firm values, because of their failure to naturally produce SN explosions (e.g., Woosley \& Weaver 1995). The very sparse observational data suggest that the Fe mass produced remains fairly constant with increasing progenitor mass, with considerable scatter (e.g., Iwamoto et al. 1998; Turatto et al. 1998; see the discussion in Nakamura et al. 1999, and in particular their Fig. 14, which shows the Fe mass produced in a number of SNe as a function of progenitor mass). The Fe yields may even be a function of stellar properties other than the initial mass.

In our model, we use two sets of SN yields, those adopted by Tsujimoto et al. (1995) and those given by case C of Woosley \& Weaver (1995). These particular yield predictions were selected because they provide rather small changes of the abundance ratio $\mathrm{Fe} / \mathrm{Mg}$ in the $\mathrm{SN}$ ejecta with stellar masses (and thus would be expected to agree fairly well with the observational results mentioned above); they then predict a smaller cloud-to-cloud scatter in the expected abundances for a given number of polluting $\mathrm{SNe}$, with respect to other yield predictions (e.g., cases A and B of Woosley \& Weaver). These models thus permit a smaller number of SNe to match the observed scatter than do other nucleosynthetic predictions: adoption of different recipes would, in general, lead to a larger predicted number of $\mathrm{SNe}$ contributing, strengthening our conclusions.

According to Tsujimoto et al. (1995), the Fe yield decreases by only a factor of 2 over a progenitor mass range from 13 to $70 M_{\odot}$ (we adopted an upper mass limit of 100 $M_{\odot}$ in this case). According to Woosley \& Weaver's case C, it rises by about a factor of 5 between 11 and $40 M_{\odot}$ (in this case, the upper mass limit was set at $50 M_{\odot}$ ). These two predictions roughly bracket the available data (see Nakamura et al. 1999). Using these yield predictions, the scatter in the yield ratios between $\mathrm{Fe}$ and the $\alpha$-elements is mainly due to the large increase in the production of the latter with masses over the same range (a factor between 50 and 100 for $\mathrm{O}$ and $\mathrm{Mg}$ ), a reasonably sound prediction of the presupernova models. Hence, according to these models, a large fraction of $\mathrm{O}$ and $\mathrm{Mg}$ are produced by few $\mathrm{SNe}$ with very massive progenitors, while $\mathrm{Fe}$ is mainly produced by the less massive $\mathrm{SNe}$ (because they are much more numerous). A random extraction over a small number of SNe may then easily produce a large scatter in the $\mathrm{O} / \mathrm{Fe}$ and $\mathrm{Mg} / \mathrm{Fe}$ ratios. 
In our simulations, we considered cases with initial masses in stars of $10^{3}$ and $10^{4} M_{\odot}$, respectively (these may be interpreted as the masses of the first forming cluster/ association). ${ }^{16}$ Assuming that all stars with masses larger than $10 M_{\odot}$ explode as $\mathrm{SNe}$, we expect respectively 3.4 and $34 \mathrm{SNe}$ in the two cases, with our choice of IMF. We then carried out $10^{5}$ and $10^{4}$ trials, respectively, for the two cases of SNe distributed according to the Scalo IMF and computed the total masses of $\mathrm{Fe}$ and $\mathrm{Mg}$ produced by these $\mathrm{SNe}$ for each trial. The rms scatter of the $[\mathrm{Mg} / \mathrm{Fe}]$ ratios from these sets are 0.43 and 0.14 dex, respectively, in the two cases when the Tsujimoto et al. yields were used (the scaling between these two values agrees well with the larger number of $\mathrm{SNe}$ of the second case). As expected, the scatter is smaller when the Woosley \& Weaver case $C$ yields are used: in these cases, we obtain rms values of 0.29 and $0.08 \mathrm{dex}$, respectively.

When we compare the predictions of this simple model with observations, we derive the typical number of SNe contributing to the ISM from which the observed stars formed as $\sim 18$ at $[\mathrm{Fe} / \mathrm{H}]=-3$ dex, and $\sim 26$ at $[\mathrm{Fe} / \mathrm{H}]=-2$ dex, when the Tsujimoto et al. yields are used. The corresponding values when Woosley \& Weaver case $\mathrm{C}$ yields are used are 7 and 10. The mass in Fe produced by $\sim 20 \mathrm{SNe}$ randomly extracted using the Scalo IMF and the Tsujimoto et al. yields is $\sim 2 M_{\odot}$; in the case of seven $\mathrm{SNe}$ with Woosley \& Weaver case $\mathrm{C}$ models, it is $\sim 0.2 M_{\odot}$. If the $\mathrm{SN}$ ejecta are used to raise the metallicity of a cloud up to $[\mathrm{Fe} / \mathrm{H}]=-3$ dex, the total original (baryonic) mass of each of the clouds is $\sim 10^{6} M_{\odot}$ in the first case, and 10 times less in the second one. These values agree fairly well with the expected Jeans mass at this epoch, and with typical values for (present-day) dwarf spheroidal galaxies and globular clusters. We intend to explore the connection between globular clusters and field stars in a future paper. With the caveats discussed above kept in mind, the value we have derived might be considered to be the characteristic mass of protogalactic fragments. Note that the typical mass of the hypothetical primordial clusters/associations are in all cases on the order of a few thousand solar masses; it is difficult for such small objects (if they really existed) to remain bound after the violent mass loss that probably occurred during their early phases.

The typical number of SNe contributing to metal enrichment of EMP stars that we obtain seems quite large, with respect to the usual assumption that metals in these stars were produced out of material polluted by ejecta from very few, possibly only one, SNe. Note that the requirement of a very small number of $\mathrm{SNe}$ (the basic ingredient of stochastic models of metal enrichment) mainly comes from the large observed scatter in elements produced by neutron-capture processes. Our result is a consequence of the relatively small scatter observed for the $[\mathrm{Mg} / \mathrm{Fe}]$ ratio, and of the assumptions about the SN yields and the IMF. A considerable reduction in the scatter predicted by these models could probably be obtained by limiting the mass range of the $\mathrm{IMF}$, because $\mathrm{SN}$ models predict a dependence of the $\mathrm{Mg}$ / Fe ratio on progenitor mass. A smaller mass range could in principle be understood if, for example, star formation in the remaining part of the cloud was very fast and only the

\footnotetext{
16 These values were considered because the resulting expected rms scatter brackets the observed values for $\mathrm{Mg} / \mathrm{Fe}$.
}

most massive stars of the first generation could evolve rapidly enough to contribute to the pollution of the ISM. However, this does not seem a palatable explanation for various reasons:

1. The naive expectation is that the mass range should be limited to the most massive SNe. On the basis of nucleosynthetic predictions, we expect that these $\mathrm{SNe}$ would produce an $[\mathrm{Mg} / \mathrm{Fe}]$ much larger than the average over the entire relevant mass range. This average value should be more appropriate for the most metal-rich halo and thick disk stars. We would then expect to observe in EMP stars an $\mathrm{Mg} / \mathrm{Fe}$ ratio much higher than in most metal-rich halo stars: however, Figure 6 shows that the $\mathrm{Mg} / \mathrm{Fe}$ ratio in EMP stars is similar to that observed in more metal-rich objects.

2. The previous consideration forces us to limit the mass range to those $\mathrm{SNe}$ that produce $[\mathrm{Mg} / \mathrm{Fe}]$ values close to the average, that is, $\mathrm{SNe}$ of about $20 M_{\odot}$. We are not aware of any simple physical explanation favoring this mass range.

3 . In order not to further increase the predicted scatter, masses for different clouds should be assumed to be similar. This might possibly be justified because the mass of these clouds is indeed of the order of magnitude of the Jeans mass; however, it should be recalled that for the adopted IMF, the number of SNe predicted for a typical Jeans mass is over 10.

4. Finally, even more detailed models such as those of Argast et al. (2000) show a scatter in the $[\mathrm{Mg} / \mathrm{Fe}]$ ratios much larger than given by observations. This demonstrates that our result is robust and is not due to the way we modeled the early process of chemical enrichment, but rather to the assumptions made about the details of the closed boxes, yields, and the IMF.

There are at least two alternative scenarios to explain the surprisingly small observed scatter in the $[\mathrm{Mg} / \mathrm{Fe}]$ ratio among EMP stars, without invoking changes in the yields, and still saving the basic concept of the stochastic model. First, it might be assumed that a generation of very massive ( $>100 M_{\odot}$ ) stars polluted the medium before the formation of the EMP stars (Qian \& Wasserburg 2001a, 2001b). Abel, Bryan, \& Norman (2002) present a fully self-consistent three-dimensional hydrodynamic simulation of the formation of one of the first stars in the universe. This is an interesting hypothesis, because the introduction of a new actor only playing at very low metallicities (and without a corresponding low-mass population, given the peculiar topheavy IMF that should be appropriate at zero metals; Oh et al. 2001) might help to explain the changes of trend/scatter in several abundance ratios observed in EMP stars, without perhaps violating other observational constraints. A full discussion is beyond the present paper; however, we wish to note here a few difficulties within this scenario that should be resolved before this hypothesis can be definitively adopted:

1. Current nucleosynthetic predictions for pair-instability $\mathrm{SNe}$ resulting from the evolution of VMSs (Heger \& Woosley 2002) do not match well the observed abundance patterns in EMP stars (see also Umeda \& Nomoto 2002). Leaving aside the more uncertain aspects, such as the normalization of the production of Fe-peak to $\alpha$-elements, the main difficulties concern the large predicted overproduction of $\mathrm{Mg}$ and $\mathrm{Si}$ with respect to $\mathrm{O}$, the odd-even pattern for $\mathrm{Fe}$ peak elements (e.g., we expect a solar $\mathrm{Cr} / \mathrm{Fe}$ ratio, at variance with observations), and the absence of production of 
elements heavier than $\mathrm{Ni}$ (such as $\mathrm{Zn}$, which is clearly overabundant in most metal-poor stars). As discussed by Umeda \& Nomoto (2002), observations concerning all these features are better explained by nucleosynthesis by the most massive core-collapse SNe. These difficulties led Qian \& Wasserburg (2002) to abandon theoretical predictions for nucleosynthetic yields for VMSs and to rely instead on empirical estimates, based on the observed abundance pattern in the EMP stars themselves.

2. As noted by Qian \& Wasserburg (2002), the concern about the small observed scatter in some abundance ratios is even greater when this scenario is adopted. In order to overcome it, either a very narrow mass range must be adopted for VMSs or the number of VMSs required should be large (the same conclusion to which we were forced when considering Type II SNe). Given the much larger yields per explosion in the case of VMSs, this last solution would create difficulties with the total observed amount of $\mathrm{Mg}$ and $\mathrm{Si}$ observed in EMP stars: in fact, a single VMS should produce enough $\mathrm{Mg}$ and $\mathrm{Si}$ to justify the total mass of $\mathrm{Mg}$ and Si present in EMP stars over the whole Galaxy (if the yields of Heger \& Woosley 2002 are used), and it seems difficult to conceive that this material could be distributed over such a large volume (but see Abel et al. 2002).

Alternatively, we may relax the closed-box approximation. In fact, there probably was some mass exchange between different fragments; in particular, we should expect selective mass loss through metal-rich winds from each fragment (the fragments were probably not able to retain within themselves all the ejecta of the SNe). Furthermore, different fragments might have formed stars at different epochs. It is then easy to imagine that a fraction of the very metal-poor fragments were probably polluted not by the ejecta of SN explosions of massive stars (or even VMSs) that formed within the fragments, but rather from material coming from other (probably much more massive) fragments that had a faster evolution. It is also possible to speculate that these larger fragments having a faster evolution do not properly belong to the halo, but rather to the material that subsequently formed the bulge or even part of the thick disk. The low metallicity of some of the EMP stars would in this case be simply the result of dilution.

Another way of stating the above is that the number of $\mathrm{SNe}$ we deduce represents some average over a wide distribution of values: in some cases only one $\mathrm{SN}$ contributed (this is required to produce, e.g., the stars with very large abundances of $r$-process elements), while in other cases there were many of them ( $>10$, and possibly even more than 100). This substantially modifies the distribution of $[\mathrm{Mg} / \mathrm{Fe}]$ values from that given by a population of clouds all of which have the same size.

If a large enough number of stars are observed with sufficiently high-quality data (and treated with a uniform abundance analysis), we might even try to verify this scenario. The distribution of $[\mathrm{Mg} / \mathrm{Fe}]$ predicted from this scenario should be very different from that given by one composed of closed boxes: there should be a compact core, due to stars formed either in very massive clouds or in clouds that received their metals from outside, and a much broader "haze" due to those stars formed in smaller, self-polluted clouds. Clearly, much larger samples of EMP stars and better calculations of $\mathrm{SN}$ yields are needed for significant progress in constraining these ideas.

\subsection{The CH star HE 0024-2523: A Very Peculiar Object}

One of the stars in our sample falls in the region of the $[\mathrm{Ba} / \mathrm{Fe}]$ versus $[\mathrm{Fe} / \mathrm{H}]$ plane populated by objects with very high $\mathrm{Ba}$ abundances. We note that this star $(\mathrm{HE}$ 0024-2523) clearly shows a $\mathrm{G}$ band of $\mathrm{CH}$ between 4300 and $4325 \AA$ ( $Q$ branch). The $\mathrm{G}$ band is much more intense than expected, given the rather low metallicity of this star $([\mathrm{Fe} / \mathrm{H}]=-2.63 \mathrm{dex})$ and its relatively high $T_{\text {eff. }}$ A preliminary comparison with synthetic spectra for the region around the band head supports a value of the $[\mathrm{C} / \mathrm{Fe}]$ ratio of about +2.2 . This may be another case of the so-called $\mathrm{CH}$ stars (or Ba stars), often found to be in binary systems (see McClure 1997; McClure \& Woodsworth 1990). In these systems, the companion evolves faster and passes through the thermally pulsing AGB phase, producing a large amount of carbon and $s$-elements. In the subsequent evolution of the system, this enriched material is ejected onto the star we presently observe. $\mathrm{CH}$ stars are expected to occur frequently among metal-poor dwarfs (and subgiants), given the small amount of material, processed within the thermally pulsing star, required to significantly pollute the outer convective envelope of metal-poor turnoff stars.

In HE 0024-2523, europium (an almost totally $r$-process element) is not observed (we checked the $4129.7 \AA$ line), and $\mathrm{Sr}$ is enhanced ( $[\mathrm{Sr} / \mathrm{Fe}] \sim 0.5)$, while $\mathrm{Ba}$ is very strong $([\mathrm{Ba} / \mathrm{Fe}] \sim 1.7)$ : this supports the identification of this star as a $\mathrm{CH}$ star.

Moreover, in very metal-poor stars (such as the one under scrutiny) there is a paucity of seeds able to capture neutrons along the $s$-chain. As a consequence, the neutrons accumulate, and heavier and heavier elements are built, so that the whole synthesis is shifted toward the heaviest nuclei, compared with solar abundances. We can then expect a large abundance of lead in this star (Travaglio et al. 2001).

The only $\mathrm{Pb}$ line available in our spectrum is the line at $4057.815 \AA$. This line is heavily contaminated by a nearby $\mathrm{CH}$ line (see also Aoki et al. 2000). We thus compared the observed profile with the result of a full spectral synthesis (see Fig. 14). The line list used in these computations was originally taken from Kurucz (1993b) and adjusted in order to obtain a good fit to the solar flux spectrum of Kurucz, Furenlid, \& Brault (1984). To improve the $\mathrm{S} / \mathrm{N}$, the observed spectrum was smoothed with a Gaussian having $\mathrm{FWHM}=0.06 \AA$; this is much less than the intrinsic line width in the spectrum of this star, since the lines are significantly broadened by rotation (see Paper I). The lead line appears to be detected, supporting an enormous $\mathrm{Pb}$ abundance $([\mathrm{Pb} / \mathrm{Fe}] \sim 3.2)$.

There is no doubt that this is a very interesting object, and we have already reobserved this star to confirm the peculiar abundance pattern shown by this class of stars (and in particular the enormous $\mathrm{Pb}$ abundance), and to search for the expected radial velocity variations associated with binarity. A detailed analysis of this star will be discussed in a separate paper (Gratton et al. 2002).

\section{SUMMARY}

We have presented a detailed abundance analysis for eight stars (seven expected to be near the main-sequence turnoff, and one probable giant) selected as extremely metal-poor candidates from the Hamburg/ESO Survey. For comparison, we also analyzed three stars (two giants 


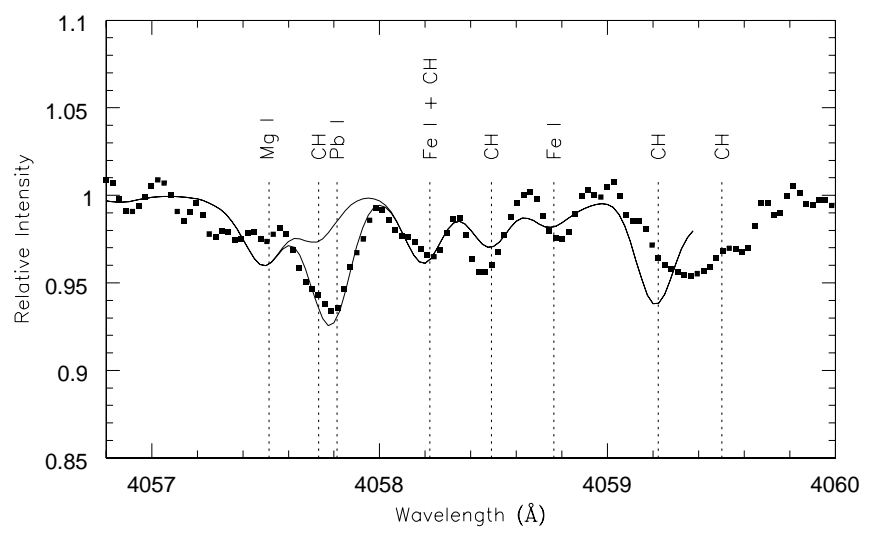

FIG. 14.-Comparison of the observed spectrum of HE 0024-2523 in the region of the $\mathrm{Pb}$ line at $4057 \AA$ and synthetic spectra computed with a low $\mathrm{Pb}$ abundance and $[\mathrm{Pb} / \mathrm{Fe}]=+3.25$. The HIRES spectrum is smoothed using a Gaussian having a FWHM of $0.05 \AA$; this smoothing does not degrade the resolution appreciably, while clearly improving the $\mathrm{S} / \mathrm{N}$.

and one dwarf) from the HK survey and three additional very bright metal-poor stars. With this work, we have doubled the number of extremely metal-poor stars $([\mathrm{Fe} / \mathrm{H}] \leq-3.0$ dex $)$ with abundance analyses based on high-precision, high spectral resolution data.

Since we have utilized stellar parameters determined independently of the spectra, the analyses of the spectra themselves yield parameters that can be used as diagnostics to test the validity of the atmospheric parameters assigned in Paper I; these appear to be valid to within the uncertainties given in Paper I. We looked for evidence of departures from LTE for Fe and did not find any, with upper limits at a level of $0.1-0.2$ dex.

We studied the $\alpha$-elements $\mathrm{Mg}, \mathrm{Si}, \mathrm{Ca}$, and $\mathrm{Ti}$; the light element $\mathrm{Al}$; the iron-peak elements $\mathrm{Sc}, \mathrm{Cr}$, and $\mathrm{Mn}$; and the neutron-capture elements $\mathrm{Sr}$ and $\mathrm{Ba}$. The first key result is that our sample of HES EMP candidates contains three stars with precision $\mathrm{Fe}$ abundances from the present highdispersion analysis with $[\mathrm{Fe} / \mathrm{H}] \leq-3.0$ dex and three more with $[\mathrm{Fe} / \mathrm{H}] \leq-2.8$ dex, and the remaining two stars are only slightly more metal-rich. Thus, the chain of procedures that led to the selection of these stars successfully produces a high fraction of extremely metal-poor stars.

In general, when we combine our sample with data from the literature, our results support the trends of element variation $[\mathrm{X} / \mathrm{Fe}]$ with decreasing $[\mathrm{Fe} / \mathrm{H}]$ found by previous investigators. These trends appear to be the same for dwarfs and for giants, extending even to the low metallicities studied here.

However, we are struck by the lack of scatter in abundance ratios among most elements in these EMP stars. While it is well known that among stars with solar metallicity, abundance ratios are essentially constant, showing only small trends with time, we naively expected stochastic effects of small numbers of SNe to have become important at the extremely low abundances we are exploring. Much to our surprise, we find that most elements have quite a small scatter $(\lessgtr 0.1 \mathrm{dex}$, some of which undoubtedly arises from experimental errors) in abundance $[\mathrm{X} / \mathrm{Fe}]$ even at $[\mathrm{Fe} / \mathrm{H}] \leq-3.0$ dex. Among the elements studied, only $\mathrm{Sr}$ and Ba show large scatter at a fixed (very low) $\mathrm{Fe} / \mathrm{H}$, and perhaps we have begun to discern genuine scatter for $\mathrm{Mn}$ and $\mathrm{Cr}$.
We discuss the implications of these results and suggest that we are almost at the point of having samples large enough to be able to constrain nucleosynthesis in the early evolution of the Galaxy and, in particular, the size of independent clouds at the time these EMP stars were formed in the Galactic halo. The preliminary value for the characteristic mass of protogalactic fragments that we deduce is tantalizingly close to that of the expected Jeans mass at this epoch, and to typical values for (present-day) dwarf spheroidal galaxies and globular clusters.

As the work of the 0Z Project advances over the next few years, we may look forward to much larger samples of EMP stars with accurate abundance determinations becoming available. The confrontation with theory raised by the small scatter in $[\mathrm{X} / \mathrm{Fe}]$ seen thus far at extremely low metallicity will become much sharper. If these small scatters persist, the assumptions normally adopted for the early chemical evolution of the Galaxy will have to be reexamined, requiring improved calculations of nuclear yields, the possibility of a previous generation of very massive stars, or mixing between "independent" primordial clouds.

Among such extremely metal-poor stars, the pollution of a stellar atmosphere with a relatively small amount of some heavy element (perhaps from a binary companion) will tend to produce a detectable abundance enhancement. As a preview of the "zoo" of peculiar stars we may thus expect to find in our future work, we note that one of the stars in our small initial sample, HE 0024-2523, was found to be a CH star, with extremely enhanced $\mathrm{Ba}$ and somewhat enhanced Sr. This main-sequence star also shows a very large overabundance of lead and appears to represent the result of the $s$-process chain operating in a very metal-poor environment.

The entire Keck/HIRES user community owes a huge debt to Jerry Nelson, Gerry Smith, Steve Vogt, and many other people who have worked to make the Keck Telescope and HIRES a reality, and who continue to operate and maintain the Keck Observatory. We are grateful to the W. M. Keck Foundation for the vision to fund the construction of the W. M. Keck Observatory. The authors wish to extend special thanks to those of Hawai'ian ancestry on whose sacred mountain we are privileged to be guests. Without their generous hospitality, none of the observations presented herein would have been possible. We thank the referee, Bruce Carney, for a very careful reading of the paper and for his constructive comments. T. C. B. acknowledges partial support for this work from grants AST 0098508 and AST 00-98549 from the National Science Foundation. This research has made use of the SIMBAD database, operated at CDS, Strasbourg, France.

\section{APPENDIX}

\section{COMPARISON OF OUR ADOPTED $g f$-VALUES WITH THOSE OF NIST}

We compare the $g f$-values we have adopted in the present work, which have been assembled from the sources listed at the end of Table 2, with those of the National Institute of Standards and Technology. The NIST Atomic Spectra Database, version 2.0 (release date 1999 March; NIST 
TABLE 8

A Comparison of Our Adopted $g f$-Values with THOSE OF NIST

\begin{tabular}{|c|c|c|c|}
\hline Ion & Lines & $\begin{array}{c}\langle g f(\text { NIST })-g f(\text { us })\rangle \\
(\text { dex })\end{array}$ & $\begin{array}{c}\sigma \\
(\mathrm{dex})\end{array}$ \\
\hline \multirow[t]{2}{*}{$\mathrm{Al}$ I............... } & 2 & 0.00 & 0.00 \\
\hline & $3^{\mathrm{a}}$ & +0.10 & 0.17 \\
\hline Mg I ....... & $2^{\mathrm{b}}$ & 0.00 & 0.00 \\
\hline Si I.......... & 1 & 0.00 & \\
\hline $\mathrm{Ca} \mathrm{I} \mathrm{........}$ & 6 & 0.00 & 0.00 \\
\hline Sc II ............. & 4 & +0.01 & 0.01 \\
\hline Ti I ........... & 16 & -0.01 & 0.02 \\
\hline Ti II ........ & 26 & -0.01 & 0.14 \\
\hline Cr I......... & 14 & 0.00 & 0.01 \\
\hline Mn I ....... & 5 & 0.00 & 0.00 \\
\hline Fe I......... & 59 & -0.01 & 0.07 \\
\hline Fe II........ & 12 & -0.01 & 0.11 \\
\hline Sr II ........ & 2 & -0.02 & 0.00 \\
\hline Ba II ........ & 1 & 0.00 & $\ldots$ \\
\hline
\end{tabular}

a There are two additional observed lines of $\mathrm{Mg}_{\mathrm{I}}$ for which there is no lifetime in either the current NIST database or in Wiese, Smith, \& Miles 1969. The NIST team is currently updating the entries for this ion.

${ }^{\mathrm{b}}$ Here we omit the $4703 \AA$ line of Mg I.

Standard Reference Database No. 78$),{ }^{17}$ provides access to critically evaluated data on atomic energy levels, wavelengths, and transition probabilities that are reasonably up to date.

For each ion that we consider, Table 8 gives the number of lines in common with the NIST database from the set given in Tables 2 and 3 and then the mean of the values of $\Delta[g f($ NIST $)-g f($ us $)]$, as well as the dispersion of the differences about the mean. In general, the results are quite satisfactory. The mean difference ranges from -0.02 to $0.00 \mathrm{dex}$. For most of the ions considered here, the dispersion of the differences is small. However, in a few cases (Mg I, Ti II, and $\mathrm{Fe}$ II) the dispersions are larger and are discussed below.

\footnotetext{
${ }^{17}$ See http://physics.nist.gov/cgi-bin/AtData/main_asd.
}

Table 8 serves to remind us that $g f$-values are still uncertain and that systematic errors of normalization exist between the results of different teams at the level of $\sim 0.1$ dex, with smaller internal uncertainties within each data set.

\section{A1. $\mathrm{Mg} \mathrm{I}$}

The present status of the oscillator strengths for $\mathrm{Mg} \mathrm{I}$ is not very good, and oscillator strengths for $\mathrm{Mg}$ I lines are generally derived from theoretical calculations. Those used by most authors are from Froese-Fischer (1975) and are used in the solar analysis of Lambert \& Luck (1978) as well. However, these are not the most recent ones. The latest updated computations are given by Mendoza \& Zeippen (1987) and by the Opacity Project group (TOP; results available through CDS). Since the Opacity Project group includes C. Mendoza, we view the TOP $g f$-values for $\mathrm{Mg}$ I as updates of the Mendoza \& Zeippen calculations. Values from the Opacity Project group agree fairly well with those from Froese-Fischer. The VALD database uses data from Kurucz CD-ROM 18 (Kurucz 1993b).

A comparison between different sources for the relevant transitions of $\mathrm{Mg}$ I is given in Table 9. Kurucz $g f$ 's agree well with the TOP calculations for the triplet lines, while they are lower by $0.2-0.3$ dex for the singlet lines.

The reason we prefer to use Kurucz $g f$ 's (those in the VALD database) is that (in other parallel studies currently in progress) with these $g f$ 's we obtain better agreement among the $\mathrm{Mg}$ I lines in well-studied moderately metal-poor stars $(-2<[\mathrm{Fe} / \mathrm{H}]<-0.5)$ with weaker $\mathrm{Mg}$ I lines (at 6318 $\AA$, etc.), those generally used in solar abundance analyses.

\section{A2. Ti II}

For $\mathrm{Ti}$ II, whenever possible we adopted experimental $g f$ 's from Bizzarri et al. (1993). These were obtained by combining branching ratios from hollow-cathode measurements with lifetimes from selective laser-induced excitation. For most lines, they are accurate within about $10 \%$. For the remaining lines, $g f$-values were taken from Magain (1985), who discussed literature values available at that epoch. When neither of these were available, we adopted those from Kurucz CD-ROM 18.

TABLE 9

Comparison of Mg i Transition Probabilities

\begin{tabular}{ccccccc}
\hline \hline Transition & FF75 & TOP & MZ & VALD $=$ Kurucz & CD-ROM $18^{\mathrm{c}}$ & Adopted \\
\hline Singlet: & & & & & & \\
$3 p^{1} P-4 d^{1} D \ldots \ldots$. & 5528 & -0.35 & -0.40 & -0.489 & -0.620 & $\ldots$ \\
$-5 d^{1} D \ldots \ldots$. & 4703 & -0.38 & -0.42 & -0.462 & -0.666 & -0.67 \\
$-7 d^{1} D \ldots \ldots$. & 4167 & -0.71 & -0.75 & -0.757 & -1.004 & -1.00 \\
$-8 d^{1} D \ldots \ldots$. & 4057 & -0.89 & -0.91 & $\ldots$ & -1.201 & -1.20 \\
$3 p^{1} P-5 s^{1} S \ldots \ldots$. & 5711 & $\ldots$ & -1.75 & -1.724 & -1.833 & $\ldots$ \\
$-6 s^{1} S \ldots \ldots \ldots$ & 4730 & $\ldots$ & -2.34 & -2.268 & -2.523 & $\ldots$ \\
Triplet: & & & & & & \\
$3 p^{3} P-4 s^{3} S \ldots \ldots$. & $5178^{\mathrm{d}}$ & $\ldots$ & 0.09 & -0.018 & 0.061 & $\ldots$ \\
& 5167 & $\ldots$ & -1.00 & -1.030 & $\ldots$ & $\ldots$ \\
& 5172 & $\ldots$ & -0.37 & -0.402 & $\ldots$ & -0.38 \\
& 5183 & $\ldots$ & -0.15 & -0.180 & $\ldots$ & -0.16 \\
\hline
\end{tabular}

a Froese-Fischer 1975.

b Kurucz 1993b.

c Mendoza \& Zeippen 1987.

d Total. 
When compared with values from the NIST database, these $g f$ 's show significant scatter (although the average values agree). The NIST $g f$ 's for Ti II are mostly from the Martin, Fuhr, \& Wiese (1988) compilation, with a few additions from Kurucz's CD-ROM 18. They do not include the most recent experimental values. When used in our abundance analysis for the three bright comparison stars, they produced a somewhat larger line-to-line scatter in the abundances.

Very recently (after our analysis was completed), Pickering, Thorne, \& Perez (2001) presented a new set of experimental $g f$ 's, based on new branching ratios from hollowcathode measurements coupled with experimental lifetimes. Their line list is more extensive, but the individual values are less accurate than those of Bizzarri et al., with typical accuracies of $10 \%-20 \%$. When compared with the set of $g f$ 's adopted here, the mean difference is $-0.02 \pm 0.03$, with an rms value of 0.12 dex from 18 lines. The scatter is definitely larger when comparison is made with $g f$ 's from those lines in the NIST database included in our list $(-0.05 \pm 0.04$, with an rms of 0.16 dex). We conclude that the adopted $g f$ 's are to be preferred to those of the NIST database.

\section{A3. $\mathrm{Fe}$ II}

The line-to-line comparison shows quite a large scatter (but no zero-point offset) between our adopted $g f$ 's for Fe II and those from NIST. These last are those from Kurucz CD-ROM 18, which are from semiempirical calculations. Our Fe II $g f$ 's are the average of the experimental values by Heise \& Kock (1990) and Hannaford et al. (1992), of the theoretical ones by Biémont et al. (1991), and of solar $g f$ 's from Blackwell, Shallis, \& Simmons (1980; these last were increased by 0.19 dex to put them on the same scale as given by the three other sources), save for four lines missing these data, for which the Kurucz $g f$ 's were adopted. For those lines in which the two values disagree, our adopted $g f$ 's are most likely more accurate than those listed by NIST.

After completing our analysis, we became aware that two more recent papers have been published with Fe II $g f$ 's: the theoretical calculations by Raassen \& Uylings (1998) and the experimental ones by Schnabel, Kock, \& Holweger (1999). These two sources agree very well with previous determinations, but with a systematic offset of $0.10 \mathrm{dex}$ in the $g f$ 's by Raassen \& Uylings. Since differences between our adopted $g f$ 's and those from these two last sources are very small $(<0.05$ dex, once Raassen \& Uylings $g f$ 's are put on the same scale as those from the other authors), save for one line, ${ }^{18}$ it was not deemed necessary to repeat our analysis.

${ }^{18}$ For the Fe I line at $4233.17 \AA$, Kurucz gives $g f=-2.00$, which we adopt in our analysis, while the value given by Schnabel et al. is -1.81 .
Abel, T., Bryan, G. L., \& Norman, M. L. 2002, Science, 295, 93

Aoki, W., Norris, J. E., Ryan, S. G., Beers, T. C., \& Ando, H. 2000, ApJ, 536, L97

Argast, D., Samland, M., Gerhard, O. E., \& Thielemann, F.-K. 2000, A\&A, 356, 873

Arpigny, C., \& Magain, P. 1983, A\&A, 127, L7

Asplund, M., \& García Pérez, A. E. 2001, A\&A, 372, 601

Asplund, M., Nordlund, A, Trampedach, R., Allende Prieto, C., \& Stein, R. F. 2000, A\&A, 359, 729

Asplund, M., Nordlund, A., Trampedach, R., \& Stein, R. F. 1999, A\&A, 346, L17

Baumüller, D., \& Gehren, T. 1997, A\&A, 325, 1088

Beers, T. C., Flynn, K., \& Gebhardt, K. 1990, AJ, 100, 32

Beers, T. C., Preston, G. W., \& Shectman, S. A. 1985, AJ, 90, 2089 .1992, AJ, 103, 1987

Biémont, E., Baudoux, M., Kurucz, R. L., Ansbacher, W., \& Pinnington, E. H. 1991, A\&A, 249, 539

Bizzarri, A., Huber, M. C. E., Noels, A., Grevesse, N., Bergeson, S. D., Tsekeris, P., \& Lawler, J. E. 1993, A\&A, 273, 707

Blackwell, D. E., Shallis, M. J., \& Simmons, G. J. 1980, A\&A, 81, 340

Booth, A. J., Shallis, M. J., \& Wells, M. 1983, MNRAS, 205, 191

Carney, B. W., Wright, J. S., Sneden, C., Laird, J. B., Aguilar, L. A., \& Latham, D. W. 1997, AJ, 114, 363

Carretta, E., Gratton, R. G., Clementini, G., \& Fusi Pecci, F. 2000, ApJ, 533,215

Castelli, F., Gratton, R. G., \& Kurucz, R. L. 1997, A\&A, 318, 841 (erratum $324,432)$

Cayrel, R. 1989, in IAU Symp. 134, The Impact of Very High S/N Spectroscopy on Stellar Physics, ed. G. Cayrel de Strobel \& M. Spite (Dordrecht: Kluwer), 345

Cayrel, R., et al. 2001a, Nature, 409, 691

2001b, in ASP Conf. Ser. 245, Astrophysical Ages and Time Scales, ed. T. von Hippel, C. Simpson, \& N. Manset (San Francisco: ASP), 244

Cayrel de Strobel, G., Soubiran, C., \& Ralite, N. 2001, A\&A, 373, 159

Christlieb, N., Green, P. J., Wisotzki, L., \& Reimers, D. 2001a, A\&A, 375, 366

Christlieb, N., Wisotzki, L., Reimers, D., Homeier, D., Koester, D., \& Heber, U. 2001b, A\&A, 366, 898

Cleveland, W. S. 1979, J. Am. Stat. Assoc., 74, 829

. 1994, The Elements of Graphing Data (rev. ed.; Summit, NJ: Hobart)

Cohen, J. G., Christlieb, N., Beers, T. C., Gratton, R., \& Carretta, E. 2002, AJ, 124, 470 (Paper I)

Froese-Fischer, C. 1975, Canadian J. Phys., 53, 184

Gehren, T., Butler, K., Mashonkina, L., Reetz, J., \& Shi, J. 2001, A\&A, 366,981

Gilroy, K. K., Sneden, C., Pilachowski, C. A., \& Cowan, J. J. 1988, ApJ, 327,298

\section{EFERENCES}

Gratton, R. G. 1989, A\&A, 208, 171

Gratton, R. G., et al. 2001, A\&A, 369, 87

. 2002, in preparation

Gratton, R. G., Carretta, E., Eriksson, K., \& Gustafsson, B. 1999, A\&A, 350,955

Gratton, R. G., \& Sneden, C. 1987, A\&A, 178, 179

. 1988, A\&A, 204, 193

1991, A\&A, 241, 501

1994, A\&A, 287, 927

Grevesse, N., \& Sauval, A. J. 1999, A\&A, 347, 348

Gustafsson, B., Bell, R. A., Eriksson, K., \& Nordlund, A. 1975, A\&A, 42, 407

Hannaford, P., Lowe, R. M., Grevesse, N., \& Noels, A. 1992, A\&A, 259, 301

Heger, A., \& Woosley, S. E. 2002, ApJ, 567, 532

Heise, C., \& Kock, M. 1990, A\&A, 230, 244

Houdashelt, M. L., Bell, R. A., \& Sweigart, A. V. 2000, AJ, 119, 1448

Iwamoto, K., et al. 1998, Nature, 395, 672

Karlsson, T., \& Gustafsson, B. 2001, A\&A, 379, 461

King, J. R. 1997, AJ, 113, 2302

Korn, A. J., \& Gehren, T. 2001, in ASP Conf. Ser. 228, Dynamics of

Star Clusters and the Milky Way, ed. S. Deiters, B. Fuchs, A. Just, R. Spurzem, \& R. Wielen (San Francisco: ASP), 494

Kurucz, R. L. 1993a, CD-ROM 13, ATLAS9 Stellar Atmosphere Programs and $2 \mathrm{~km} / \mathrm{s}$ Grid (Cambridge: Smithsonian Astrophys. Obs.)

. 1993b, CD-ROM 18, SYNTHE Spectrum Synthesis Programs and Line Data (Cambridge: Smithsonian Astrophys. Obs.)

Kurucz, R. L., Furenlid, I., \& Brault, J. 1984, Solar Flux Atlas from 296 to $1300 \mathrm{~nm}$ (Sunspot, NM: Natl. Sol. Obs.)

Lambert, D. L., \& Luck, R. E. 1978, MNRAS, 183, 79

Magain, P. 1984, A\&A, 134, 189

1985, A\&A, 146, 95

Martin, G. A., Fuhr, J. R., \& Wiese, W. L. 1988, Atomic Transition Probabilities Scandium through Manganese (J. Phys. Chem. Ref. Data, 17, suppl. 3) (New York: Natl. Bur. Stand.)

McClure, R. D. 1997, PASP, 109, 536

McClure, R. D., \& Woodsworth, A. W. 1990, ApJ, 352, 709

McWilliam, A. 1997, ARA\&A, 35, 503

. 1998, AJ, 115, 1640

McWilliam, A., Preston, G. W., Sneden, C., \& Searle, L. 1995, AJ, 109, 2757

Mendoza, C., \& Zeippen, C. J. 1987, A\&A, 179, 339

Miller, G. E., \& Scalo, J. M. 1979, ApJS, 41, 513

Nakamura, T., Umeda, H., Nomoto, K., Thielemann, F.-K., \& Burrows, A. 1999, ApJ, 517, 193

Nissen, P. E., \& Schuster, W. J. 1997, A\&A, 326, 751

Norris, J. E., Beers, T. C., \& Ryan, S. G. 2000, ApJ, 540, 456

Norris, J. E., Ryan, S. G., \& Beers, T. C. 2001, ApJ, 561, 1034 (NRB01) 
Oh, S. P., Nollett, K. M., Madau, P., \& Wasserburg, G. J. 2001, ApJ, 562, L1

Pagel, B. E. J. 1997, Nucleosynthesis and Chemical Evolution of Galaxies (Cambridge: Cambridge Univ. Press)

Pickering, J. C., Thorne, A. P., \& Perez, R. 2001, ApJS, 132, 403 (erratum 138, 247 [2002])

Qian, Y.-Z., \& Wasserburg, G. J. 2001a, ApJ, 549, 337 $.2001 \mathrm{~b}$, ApJ, 559, 925
-2002, ApJ 567,515

Raassen, A. J. J., \& Uylings, P. H. M. 1998, A\&A, 340, 300

Rutten, R. J., \& van der Zalm, E. B. J. 1984, A\&AS, 55, 143

Ryan, S. G., Norris, J. E., \& Beers, T. C. 1996, ApJ, 471, 254 (RNB96)

Ryan, S. G., Norris, J. E., \& Bessell, M. S. 1991, AJ, 102, 303

Schlegel, D. J., Finkbeiner, D. P., \& Davis, M. 1998, ApJ, 500, 525

Schnabel, R., Kock, M., \& Holweger, H. 1999, A\&A, 342, 610

Simmons, J., \& Blackwell, D. E. 1982, A\&A, 112, 209

Sneden, C., Cowan, J. J., Ivans, I. I., Fuller, G. M., Burles, S., Beers, T. C., \& Lawler, J. E. 2000, ApJ, 533, L139

Steffen, M. 1985, A\&AS, 59, 403

Stephens, A. 1999, AJ, 117, 1771

Thévenin, F., \& Idiart, T. P. 1999, ApJ, 521, 753
Timmes, F. X., Woosley, S. E., \& Weaver, T. A. 1995, ApJS, 98, 617

Toenjes, R., Schatz, H., Kratz, K.-L., Pfeiffer, B., Beers, T. C., Cowan, J., \& Hill, V. 2001, in ASP Conf. Ser. 245, Astrophysical Ages and Time Scales, ed. T. von Hippel, C. Simpson, \& N. Manset (San Francisco: ASP), 376

Travaglio, C., Gallino, R., Busso, M., \& Gratton, R. 2001, ApJ, 549, 346

Tsujimoto, T., Nomoto, K., Yoshii, Y., Hashimoto, M., Yanagida, S., \& Thielemann, F.-K. 1995, MNRAS, 277, 945

Turatto, M., et al. 1998, ApJ, 498, L129

Umeda, H., \& Nomoto, K. 2002, ApJ, 565, 385

Vogt, S. E., et al. 1994, Proc. SPIE, 2198, 362

Wasserburg, G. J., \& Qian, Y.-Z. 2000, ApJ, 529, L2

Wiese, W. L., Smith, M. W. \& Miles, B. M. 1969, Atomic Transition Probabilities, Vol. 2: Sodium through Calcium (Natl. Stand. Ref. Data Ser. 22) (Washington: GPO)

Wisotzki, L., Köhler, T., Groote, D., \& Reimers, D. 1996, A\&AS, 115, 227

Woosley, S. E., \& Weaver, T. A. 1995, ApJS, 101, 181

Yi, S., Demarque, P., Kim, Y.-C., Lee, Y.-W., Ree, C. H., Lejeune, T., \& Barnes, S. 2001, ApJS, 136, 417

Zacharias, N., et al. 2000, AJ, 120, 2131

Zhao, G., \& Magain, P. 1990, A\&AS, 86, 85 